BNL-114102-2017-JA

\title{
Understanding the Effect of Preparative Approaches in the Formation of "Flowerlike"Li4Ti5O12 - Multiwalled Carbon Nanotube Composite Motifs with Performance as High-rate Anode Materials for Li-ion Battery Applications
}

Lei Wang, Yiman Zhang, Coray McBean, Megan E. Scofield, Jiefu Yin, Amy C. Marschilok, Kenneth J. Takeuchi, Esther S. Takeuchi, and Stanislaus S. Wong

Submitted to the Journal of the Electrochemical Society

January 1, 2017

Energy and Photon Sciences Directorate

Brookhaven National Laboratory

\author{
U.S. Department of Energy \\ USDOE Office of Science (SC), \\ Basic Energy Sciences (BES) (SC-22)
}

Notice: This manuscript has been co-authored by employees of Brookhaven Science Associates, LLC under Contract No. DE-SC0012704 with the U.S. Department of Energy. The publisher by accepting the manuscript for publication acknowledges that the United States Government retains a non-exclusive, paid-up, irrevocable, world-wide license to publish or reproduce the published form of this manuscript, or allow others to do so, for United States Government purposes. 


\section{DISCLAIMER}

This report was prepared as an account of work sponsored by an agency of the United States Government. Neither the United States Government nor any agency thereof, nor any of their employees, nor any of their contractors, subcontractors, or their employees, makes any warranty, express or implied, or assumes any legal liability or responsibility for the accuracy, completeness, or any third party's use or the results of such use of any information, apparatus, product, or process disclosed, or represents that its use would not infringe privately owned rights. Reference herein to any specific commercial product, process, or service by trade name, trademark, manufacturer, or otherwise, does not necessarily constitute or imply its endorsement, recommendation, or favoring by the United States Government or any agency thereof or its contractors or subcontractors. The views and opinions of authors expressed herein do not necessarily state or reflect those of the United States Government or any agency thereof. 
Understanding the Effect of Preparative Approaches in the Formation of "Flowerlike" $\mathrm{Li}_{4} \mathrm{Ti}_{5} \mathrm{O}_{12}$ - Multiwalled Carbon Nanotube Composite Motifs with Performance as High-rate Anode Materials for Li-ion Battery Applications

Lei Wang ${ }^{1}$, Yiman Zhang $^{1}$, Coray McBean ${ }^{1}$, Megan E. Scofield ${ }^{1}$, Jiefu Yin ${ }^{1}$, Amy C.

Marschilok $^{1,2}$, Kenneth J. Takeuchi ${ }^{1,2}$, Esther S. Takeuchi ${ }^{1,2,3}$, and Stanislaus S. Wong ${ }^{1,4, *}$

Email: stanislaus.wong@,stonybrook.edu; sswong@bnl.gov

${ }^{1}$ Department of Chemistry, State University of New York at Stony Brook,

Stony Brook, NY 11794-3400

${ }^{2}$ Department of Materials and Engineering, State University of New York at Stony Brook,

Stony Brook, NY 11794-2275

${ }^{3}$ Energy Sciences Directorate, Interdisciplinary Sciences Building, Building 734,

Brookhaven National Laboratory, Upton, NY 11973

${ }^{4}$ Condensed Matter Physics and Materials Sciences Division, Brookhaven National Laboratory, Building 480, Upton, NY 11973

Keywords: Lithium titanate; multiwalled carbon nanotubes; high rate Li-ion batteries; nanoscale synthesis; structure-property correlations 


\begin{abstract}
Herein we highlight the significance of nanoscale attachment modality as an important determinant of observed electrochemical performance. Specifically, controlled loading ratios of multi-walled carbon nanotubes (MWNTs) have been successfully anchored onto the surfaces of a unique "flower-like" $\mathrm{Li}_{4} \mathrm{Ti}_{5} \mathrm{O}_{12}$ (LTO) micro-scale sphere motif, for the first time, using a number of different and distinctive preparative approaches, including (i) a sonication method, (ii) an in situ direct-deposition approach, (iii) a covalent attachment protocol, as well as (iv) a $\pi$ - $\pi$ interaction strategy. In terms of structural characterization, the composites generated by physical sonication as well as non-covalent $\pi$ - $\pi$ interactions retained the intrinsic hierarchical "flower-like" morphology and exhibited a similar crystallinity profile as compared with that of pure LTO. By comparison, the composite prepared by an in situ direct deposition approach yielded not only a fragmented LTO structure, likely due to the possible interfering presence of the MWNTs themselves during the relevant hydrothermal reaction, but also a larger crystallite size, owing to the higher annealing temperature associated with its preparation. Finally, the composite created via covalent attachment was covered with an amorphous insulating linker, which probably led to a decreased contact area between the LTO and the MWNTs and hence, a lower crystallinity in the resulting composite. Electrode tests suggested that the composite generated by $\pi$ - $\pi$ interactions out-performed the other three analogous heterostructures, due to a smaller charge transfer resistance as well as a faster Li-ion diffusion. In particular, the LTO-MWNT composite, produced by $\pi-\pi$ interactions, exhibited a reproducibly high rate capability as well as a reliably solid cycling stability, delivering $132 \mathrm{~mA} \mathrm{~h} \mathrm{~g}^{-1}$ at $50 \mathrm{C}$, after 100 discharge/charge cycles, including 40 cycles at a high $(>20 \mathrm{C})$ rate. Such data denote the highest electrochemical performance measured to date as compared with any LTO-carbon nanotube-based composite materials previously reported, under high discharge rate conditions, and tangibly underscore the correlation between preparative methodology and the resulting performance metrics.
\end{abstract}




\section{Introduction}

One of the key goals of sustainability is to create reliable, efficient, and costeffective alternatives for fueling and powering conventional devices. In particular, the development of Li-ion batteries (LIBs) possessing high rate performance, superior durability, as well as desirable environmental sustainability is critical to advancing applications ranging from smart electronics to electric vehicles. ${ }^{1-3}$ Conventional LIBs associated with graphite anode materials may suffer from lithium deposition on the anode surface, leading to poor cycling stability. ${ }^{4-6}$ Hence, spinel $\mathrm{Li}_{4} \mathrm{Ti}_{5} \mathrm{O}_{12}$ (LTO) has been proposed to be a promising alternative anode material, because of the material's several intrinsic advantages. These include: (i) a high and stable potential plateau value (i.e. 1.55 V versus $\mathrm{Li} / \mathrm{Li}^{+}$), thereby not only avoiding the formation of lithium dendrites but also mitigating the formation of the solid-electrolyte-interphase via electrolyte reduction; (ii) an excellent durability over an extended cycle life due to a negligible volume change during electrochemical cycling; as well as (iii) a high thermal stability, potentially enabling their use at elevated temperatures. ${ }^{4,7}$ Hence, overcoming the inherently low conductivity $\left(<10^{-13} \mathrm{~S} \mathrm{~cm}^{-1}\right)$ coupled with the moderate lithium ion diffusion coefficient $\left(10^{-9}-10^{-13} \mathrm{~cm}^{2} \mathrm{~s}^{-1}\right)$ of bulk LTO represent achievable objectives for further improvement of electrochemical performance. ${ }^{4,8,9}$

A significant amount of effort has been expended in terms of developing strategies to ameliorate the rate performance of LTO electrodes. The first approach has been to design unique nanostructure motifs of LTO in order to enhance their electronic and Li-ion conductivity, within the context of zero-dimensional (0D) nanoparticles, ${ }^{10,11}$ one-dimensional (1D) nanowires and nanotubes, ${ }^{12,13}$ as well as three-dimensional (3D) 
structural architectures. ${ }^{5,14,15}$ Recently, our group reported on the successful generation of novel hierarchical 'flower-like' LTO microspheres, consisting of thin saw-tooth shaped constituent nanosheets that had been synthesized by (i) a facile and large-scale hydrothermal process involving recyclable precursors followed by (ii) a short, relatively low-temperature calcination process in air. We noted that in our hierarchical assemblies, the thin nanosheets gave rise to shortened Li-ion diffusion distances and enhanced contact area with electrolyte, while the micron-scale spherical assemblies themselves possessed thermodynamic stability and high tap-density. ${ }^{14,16}$ The resulting electrodes exhibited both excellent rate capabilities and stable cycling performance, delivering, as an example, as much as $137 \mathrm{mAh} \mathrm{g}^{-1}$ with a capacity retention of $\sim 87 \%$ at a discharge rate of $20 \mathrm{C}$ from cycles 101 to $300 .^{17}$

The second generalized strategy to enhance the electronic conductivity between the LTO anode material and the current collector has been through the mediation of a conductive coating onto an underlying LTO surface. ${ }^{9,18,19}$ Carbon nanotubes (CNTs) are unique and desirable as a result of their exceptional electrical conductivity and outstanding mechanical strength in part due to their anisotropic structure. As such, they may represent a particularly promising conductive additive for improving the rate capabilities of LTO composites. ${ }^{7,20,21}$ Specifically, substantial research has successfully highlighted the plausibility of incorporating CNTs within the context of $0 \mathrm{D}, 1 \mathrm{D}$, and 2D LTO-based anode materials, respectively, using either physical mixing or in situ deposition methods. ${ }^{22-27}$

Representative examples of these varied efforts are discussed. For example, Fang et al. prepared LTO/CNT-based composites by embedding submicron LTO particles 
within a network of conductive multi-walled carbon nanotubes (MWNTs); the resulting composite exhibited excellent rate and cycling performance, i.e. a value of $163 \mathrm{mAh} \mathrm{g}{ }^{-1}$ at $2 \mathrm{C}$ after 1000 cycles. ${ }^{28}$ In addition, Ni et al. reported on the use of CNTs to which LTO nanoparticles had been immobilized by means of liquid phase deposition as a composite anode material for high rate LIBs; these materials delivered $112 \mathrm{mAh} \mathrm{g}^{-1}$ at a 20 C discharge rate. ${ }^{9}$ Moreover, Shen et al. were able to grow LTO sheathes with a measured thickness of $\sim 25 \mathrm{~nm}$ on the exterior of a MWNT core, and noted that these structures exhibited significantly higher rate capabilities of 158.9, 147.8, 136.3, and 123.6 $\mathrm{mAh} \mathrm{g}^{-1}$ at rates of $1,5,10$ and $20 \mathrm{C}$, and an even better capacity retention potential, as compared with their uncoated analogues. ${ }^{29}$ Finally, Zhang et al. recently fabricated LTO nanosheet/CNT composites, yielding $145 \mathrm{mAh} \mathrm{g}^{-1}$ and $118 \mathrm{mAh} \mathrm{g}^{-1}$ at discharge rates of $11 \mathrm{C}$ and $23 \mathrm{C}$, respectively. ${ }^{27}$ However, to date, there have been few if any reports focused on the integration of CNTs with 3D hierarchical LTO motifs into a defined, discrete, and rationally designed architecture. Why hence is there an emphasis on this particular heterostructure motif?

The main reason is that a hybrid composite possesses a number of outstanding 'structural design' advantages that can assist in improving the measured anode performance. First, the thin constituent nanosheets within the flower-like LTO micronscale spheres can provide for a reduced lithium ion diffusion distance. ${ }^{30,31}$ Second, the numerous roughened surfaces of the thin petal-like nanosheets, associated with a high surface area, represent potentially favorable active sites for the interaction of the electrolyte with LTO, thereby ultimately providing for improved voltage profiles and charge/discharge dynamics. Third, the incorporation of the MWNT network not only 
efficiently provides for viable electrical pathways to the LTO flower-like structure from the current collector but also increases the mechanical stability of the underlying electrode through the interweaving of the electrode components. ${ }^{7}$

As such, herein, we have constructed novel 3D hierarchical flower-like LTOMWNT composites, for the first time, with the explicit aim of precisely tuning for and potentially optimizing the nature of the interaction of the LTO with the underlying MWNT framework. Furthermore, although prior work has been conducted in terms of generating MWNT-LTO nanocomposites to enhance electrode performance, to the best of our knowledge, there have been few if any efforts primarily focused on chemically controlling and improving upon the ion transport between the constituent LTO motifs and the adjoining CNTs in terms of systematically engineering the nature of the molecular junction between these two species through judiciously chosen attachment strategies.

Therefore, in separate experiments, our as-prepared 3D LTO microspheres have been subsequently embedded into the MWNT network through (i) physical sonication, (ii) direct in situ deposition, (iii) covalent attachment, as well as (iv) simple, non-covalent $\pi-\pi$ interaction strategies. It is worth noting that the incorporation of various linkers as connective, mediating 'bridges' between LTO and MWNTs with the goal of enhancing electrode performance for methods (iii) and (iv) had never, to the best of our knowledge, been previously reported with prior LTO-MWNT composite formation. In so doing, for the first time in these types of systems, we have sought to correlate the electrochemical performances of these individual distinctive LTO-MWNT composites with their specific attachment strategy, i.e. with the particular preparative treatment process used to generate each of these composite materials. Subsequent structural and electrochemical 
characterization has yielded valuable insights into the structural properties, the rate performance, the impact of the connective bridging linkers, and the nature of the attachment modality upon the resulting ion transport of the composite material.

\section{Experimental}

\subsection{Functionalization of MWNTs.}

Pristine MWNTs (SES Research, 95\% nanotubes and 2\% amorphous carbons), presented in Figure $\mathrm{S} 1 \mathrm{~A} \dagger$, were initially dispersed in concentrated $\mathrm{HNO}_{3}$ (Sigma-Aldrich, $70 \%$ ) by sonication and further refluxing at $120^{\circ} \mathrm{C}$ for $4 \mathrm{~h}$ in order to (a) remove any remnant metal catalysts and carbonaceous impurities (indicated by the white circles in Figure S1A), as well as to (b) generate functional, oxygenated carboxylic acid moieties onto the nanotube surfaces. The resulting purified and oxidized MWNTs (Figure S1B $\uparrow$ ), possessing a diameter range of 10 to $30 \mathrm{~nm}$, were filtered through a $200 \mathrm{~nm}$ pore diameter polycarbonate membrane (Millipore), thoroughly washed with excess water, and dried at $80^{\circ} \mathrm{C}$ for $18 \mathrm{~h}$.

\subsection{Synthesis of 'flower-like' LTO micron-scale spheres.}

The typical synthesis of our 'flower-like' LTO microspheres has been reported previously by our group. ${ }^{17}$ In brief, $\sim 40$ pieces of Ti foil (STREM chemicals, 99.7\%), consisting of $1 \mathrm{~cm} \times 1 \mathrm{~cm}$ squares, were placed in an $120 \mathrm{~mL}$ autoclave and reacted with $86.1 \mathrm{~mL}$ of $0.5 \mathrm{M} \mathrm{LiOH}$ (Acros Organics ${ }^{\mathrm{TM}}, 98 \%$ ) and $7.83 \mathrm{~mL}$ of $30 \%(\mathrm{w} / \mathrm{w}) \mathrm{H}_{2} \mathrm{O}_{2}$ (VWR) aqueous solution, followed by strong stirring at room temperature for $15 \mathrm{~min}$. Afterwards, the as-prepared mixture solution was subsequently heated at $130^{\circ} \mathrm{C}$ for $4 \mathrm{~h}$. The resulting suspension and as-formed white precipitate were separated by vacuum 
filtration, washed with aliquots of deionized water, and ultimately oven dried at $80^{\circ} \mathrm{C}$.

The final products were annealed at $500^{\circ} \mathrm{C}$ in air for $3 \mathrm{~h}$ in a muffle furnace in order to obtain 'flower-like' LTO microspheres.

\subsection{Synthesis of 'flower-like' $\mathrm{Li}_{4} \mathrm{Ti}_{5} \mathrm{O}_{12}$ micron-scale sphere - MWNT composites.}

To demonstrate the inherent flexibility of our preparative methodology and to further study the correlation between synthetic strategies, i.e. the chemistry of heterostructure formation, and electrode performance, composites were generated by four complementary strategies, namely by means of (1) a physical mixing of pre-formed structures by sonication; (2) a direct in situ deposition of MWNTs onto the underlying LTO micron-scale spheres within the context of a hydrothermal reaction environment; (3) the covalent attachment of the two constituent components through the mediation of a silane linker molecule; and (4) the formation of non-covalent $\pi$ - $\pi$ interactions stabilized with short-chain, electroactive aromatic linker molecules.

Sonication method. The acid-functionalized MWNTs were well dispersed in dimethyl sulfoxide (DMSO) by ultrasonication for $1 \mathrm{~h}$ in order to obtain a clear black solution. As-prepared LTO micron-scale spheres were sonicated in water for $30 \mathrm{~min}$ before being added into the MWNT solution in a drop-wise manner. The mixture solution was further ultra-sonicated for another $1 \mathrm{~h}$. The final product was collected by filtration, washed with deionized water, and dried at $80^{\circ} \mathrm{C}$ in order to obtain the resulting LTOMWNT composites.

In situ direct deposition approach. The functionalized MWNTs were added to the autoclave together with the precursors of lithium titanate, namely $\mathrm{H}_{2} \mathrm{O}_{2}, \mathrm{LiOH}$, and Ti foil, using the same reaction parameters, as previously described. The resulting grey 
product was further annealed at $600^{\circ} \mathrm{C}$ for $3 \mathrm{~h}$ under an $\mathrm{N}_{2}$ atmosphere in a tube furnace in order to preserve the underlying structural integrity of MWNTs.

Covalent attachment protocol. The relevant reaction procedures are depicted in Scheme 1. As-prepared LTO micron-scale spheres were initially functionalized with (3aminopropyl) triethoxysilane (APTES) linker molecules (Acros Organics, 99\%) by dispersing them in anhydrous organic DMSO solvent, in order to inhibit the formation of undesirable polysilsesquioxane that normally is generated through the hydrolytic condensation of organosilanes in either water or ethanol-water media. ${ }^{32-34}$ The coated LTO sample was subsequently reacted at $85^{\circ} \mathrm{C}$ for $18 \mathrm{~h}$ under a $\mathrm{N}_{2}$ atmosphere, followed by thermal curing at $120^{\circ} \mathrm{C}$ for $24 \mathrm{~h}$ in order to generate amine-terminated LTO. The resulting $\mathrm{NH}_{2}$-terminated LTO product was collected by centrifugation and further washed with DMSO for three times to remove any remaining, free-standing APTES molecules. The acid-functionalized MWNTs were dispersed in a $\mathrm{H}_{2} \mathrm{O}$ : DMSO mixture (i.e., a 1: 2 ratio by volume) by sonication followed by the addition of 1-ethyl-3-(3dimethylaminopropyl) carbodiimide (EDC) (Sigma-Aldrich, 99.9\%), and Nhydroxysuccinimide (NHS) (Sigma-Aldrich, 98\%) in order to activate pendant carboxylic acid groups. The APTES-derivatized LTO were dispersed in water and mixed in with the MWNT solution in 2-morpholino-ethanesulfonic acid (MES) (Sigma-Aldrich, 99.5\%) buffer solution. The resulting mixture was reacted under vigorous stirring for $24 \mathrm{~h}$. The final product was ultimately filtered, washed with excess deionized water, and finally dried at $80^{\circ} \mathrm{C}$ to obtain LTO-MWNT composites.

$\pi-\pi$ interaction strategy. The reaction details are demonstrated in Scheme 2. Assynthesized LTO spheres were initially dispersed in an ethanolic solution of 4- 
mercaptobenzoic acid (4-MBA) (Aldrich, 99\%) linker molecules. The mixture was stirred at $60^{\circ} \mathrm{C}$ for $18 \mathrm{~h}$ in order to create a sample of 4-MBA functionalized LTO, in which the terminal carboxylic acid groups of the ligand are bound onto the Ti sites localized on the LTO surface through either a monodentate or bidentate coordination mode. ${ }^{35}$ The molecular-coated LTO product was isolated by vacuum filtration and further washed with ethanol for multiple times to remove any remaining, unbound 4-MBA linkers. Such 4MBA functionalized LTO microspheres were subsequently reacted with the oxidized MWNTs, through sonication in a mixture of ethanol and DMSO (in a 3:1 volume ratio) solvents for $2 \mathrm{~h}$, in order to facilitate favorable and stabilizing non-covalent $\pi-\pi$ interactions between the phenyl rings within the aromatic, conjugated linker molecules and the underlying MWNT network. The resulting composites were subsequently vacuum filtered, washed, and ultimately oven dried at $80^{\circ} \mathrm{C}$.

\subsection{Structural Characterization.}

X-ray Diffraction (XRD). Crystallographic data on as-prepared LTO micronscale spheres as well as various LTO-MWNT composites were obtained using a Rigaku Miniflex II diffractometer, operating in the Bragg configuration using $\mathrm{Cu} \mathrm{K} \alpha$ radiation (1.54 $\AA$ ). Specifically, diffraction data were collected in a range of $10^{\circ}-70^{\circ}$ at a scanning rate of $1^{\circ}$ per minute using a zero-background holder. A Rietveld refinement has been performed on as-obtained XRD profiles using the EXPGUI interface in the Match! (Crystal Impact GbR) software, thereby allowing for phase identification from powder diffraction data.

Electron microscopy. The structure, morphology, and size of the LTO-MWNT micron-scale spheres were probed using an analytical high resolution SEM (JEOL 7600F) 
instrument, operating at an accelerating voltage range of 5 to $15 \mathrm{kV}$ and equipped with EDX capabilities. To prepare these samples for subsequent characterization, fixed quantities were dispersed in ethanol and sonicated for $\sim 1 \mathrm{~min}$, prior to their deposition onto an underlying silicon ( $\mathrm{Si}$ ) wafer. Low-magnification TEM images were collected using an accelerating voltage of $120 \mathrm{kV}$ on a JEOL JEM-1400 instrument, with a $2048 \mathrm{x}$ 2048 Gatan CCD Digital Camera. High resolution TEM (HR-TEM) images coupled with SAED patterns were recorded using a JEOL JEM-3000F microscope, equipped with a Gatan image filter (GIF) spectrometer, operating at an accelerating voltage of $300 \mathrm{kV}$. In addition, data on elemental mappings of $\mathrm{Ti}, \mathrm{O}, \mathrm{C}, \mathrm{N}, \mathrm{Si}$, and $\mathrm{S}$ within various MWNTLTO composite samples were collected using the energy dispersive spectrometer (EDS) associated with the JEOL JEM-1400 instrument.

Thermo-gravimetric Analysis (TGA). Data were acquired using a TGA Q500 instrument over a relatively broad temperature range spanning from 30 to $800^{\circ} \mathrm{C}$ under an air atmosphere, using a heating rate of $10^{\circ} \mathrm{C} / \mathrm{min}$.

FT-mid-IR. Relevant data were obtained on a Nexus 670 (Thermo Nicolet) spectrometer equipped with a single-reflectance zinc selenide (ZnSe) ATR accessory, a $\mathrm{KBr}$ beam splitter, and a DTGS KBr detector. Specifically, solid samples were placed onto a ZnSe crystal. Measurements were obtained in reflectance mode by using a Smart Performer module.

\subsection{Electrochemical Methods.}

Electrode and cell preparation. Electrodes were prepared using a slurry cast method on an aluminum metal foil with MWNT- $\mathrm{Li}_{4} \mathrm{Ti}_{5} \mathrm{O}_{12}(\mathrm{MWNT}-\mathrm{LTO})$ as the active material combined with $10 \%$ carbon and 5\% polyvinylidene fluoride (PVDF) binder. 
The active mass loading of the electrodes was $2.3 \mathrm{mg} / \mathrm{cm}^{2}$ on average wherein only the LTO mass was used for the calculation of specific capacity. Two electrode cells were assembled in an Ar-filled glovebox using lithium metal as the counter electrode, $1 \mathrm{M}$ $\mathrm{LiPF}_{6}$ in a mixture of ethylene carbonate and dimethyl carbonate (volume ratio of 3: 7) as the electrolyte, and polypropylene membranes as separators.

Electrochemical testing methods. Cyclic voltammetry $(\mathrm{CV})$ data were recorded in a two-electrode configuration using the MWNT-LTO-based electrode as the working electrode and the lithium electrode as both the auxiliary and reference electrodes. Two cycles each over a voltage range of $1.0-3.0 \mathrm{~V}$ were applied, using scan rates of $0.5,1.0$, 2.0 , and $5.0 \mathrm{mV} / \mathrm{s}$, respectively.

Electrochemical impedance spectroscopy (EIS) was recorded for the cells with a $\pm 5 \mathrm{mV}$ AC signal and a frequency range from $10^{5}$ to $1 \mathrm{~Hz}$ at a temperature of $30^{\circ} \mathrm{C}$. Data were fit to the equivalent circuit using Zview software (Scribner Associates).

The electrochemical cells were tested in galvanostatic mode using voltage limits of $1.0 \mathrm{~V}$ for discharge and 3.0 $\mathrm{V}$ for charge. Cells were discharged using a series of rates ranging from $\mathrm{C} / 2$ to $100 \mathrm{C}$, and the charge rate in all cases was $\mathrm{C} / 2$. Calculations of $\mathrm{C}$ rate were based upon a capacity value of $175 \mathrm{mAh} / \mathrm{g}$ for LTO. Capacity calculations are based upon the averages of data obtained from two cells, taking into account the mass of $\mathrm{Li}_{4} \mathrm{Ti}_{5} \mathrm{O}_{12}$ in the electrode.

\section{Results and Discussion}

\subsection{Morphology and structure of the materials}


Typical XRD patterns of (i) MWNTs, (ii) pure 'flower-like' LTO micron-scale spheres, and (iii) LTO-MWNT composites, prepared using different synthetic strategies, respectively, are presented in Figure 1 (with the MWNT loading ratio in each composite as $5 \mathrm{wt} \%$, as confirmed by the TGA profile, shown in Figure S2†). The pure LTO can be indexed to the face-centered cubic $(f c c)$ spinel structure of $\mathrm{Li}_{4} \mathrm{Ti}_{5} \mathrm{O}_{12}$ (i.e. JCPDS card No. 49-0207). The broadened peaks in the XRD pattern suggest that as-synthesized LTO possesses a relatively small crystallite size, which has been calculated to be $\sim 12.6 \mathrm{~nm}$ using the Debye-Scherrer equation.

Upon MWNT addition, it was observed that all of the diffraction peak positions of the LTO did not necessarily change, with the major diffraction peaks located at $2 \theta$ values of $18.4^{\circ}, 35.7^{\circ}, 43.3^{\circ}, 47.4^{\circ}, 57.3^{\circ}, 62.8^{\circ}$, and $66.2^{\circ}$, which could be ascribed to the (111), (311), (400), (331), (333), (440), and (531) planes, respectively, of a face-centered cubic spinel structure, possessing a $F d-3 m$ space group. A new and reasonably broadened peak centered at $26.2^{\circ}$ could be assigned to the (002) lattice plane of the MWNTs, present in the XRD profile of the MWNTs alone, as highlighted in the top panel of Figure 1. ${ }^{36}$

When comparing these data with pure LTO, we found that the LTO-MWNT composites generated by both the physical sonication as well as the non-covalent $\pi-\pi$ interaction methods yielded little if any obvious change in the degree of overall crystallinity. By contrast, the LTO-MWNT sample, prepared by in situ deposition protocol, evinced sharper diffraction peaks, suggestive of the formation of a larger crystallite size, possibly due to the higher annealing temperature used. In effect, based upon the Debye-Scherrer equation, the crystallite size derived from the composite prepared by the in situ method was calculated to be $30.0 \mathrm{~nm}$ as compared with a $12.6 \mathrm{~nm}$ 
value associated with both pure LTO as well as with the two composites generated by physical sonication and non-covalent $\pi-\pi$ interaction methods. It has been reported that the presence of a larger crystallite size may lead to a reduced rate performance of this composite material as compared with analogous composites possessing a smaller crystallite size. This assertion has been ascribed to the observation that a relatively small crystallite size often favors enhanced rate capabilities and long-term cyclability, because of a combination of not only low charge transfer impedance but also decreased $\mathrm{Li}^{+}$ diffusion impedance. ${ }^{37,38}$

Additionally, the LTO-MWNT composite, prepared by means of covalent immobilization, gave rise to less well-defined XRD peaks and lower signal-to-noise ratios, thereby indicating the presence of material with a lower degree of crystallinity, a situation which could be possibly attributed to the existence of amorphous APTES linker molecules ${ }^{39}$ on the LTO surface. The calculated crystallite size for this covalently formed composite was estimated to be $12.0 \mathrm{~nm}$, similar to that of pure LTO alone, thereby indicating that the APTES functionalization process itself had not altered the apparent crystallinity of LTO itself.

In order to gain a deeper structural understanding of the various samples formed, Rietveld refinements were conducted (Figure S3 $\uparrow$ ), in which we were able to observe variations in the measured lattice parameter, $a$. For example, pure LTO possesses a lattice parameter of $8.35 \AA$, whereas the LTO-MWNT ( $5 \mathrm{wt} \%$ ) composites generated by sonication, direct in situ, covalent attachment, and non-covalent $\pi-\pi$ interaction strategies yielded corresponding lattice parameters of $8.35 \AA, 8.36 \AA, 8.36 \AA$, and $8.36 \AA$, respectively. Interestingly, it was also noted that the composite derived from the direct in 
situ method in particular contained $0.9 \%$ rutile $\mathrm{TiO}_{2}$ and $0.8 \%$ anatase $\mathrm{TiO}_{2}$ impurities, as shown in Figure S3C $\uparrow$.

Our pure LTO micron-scale spheres exhibited a hierarchical 'flower-shape' structure with an overall diameter of $1 \mu \mathrm{m}$, as indicated by the SEM image in Figure $\mathrm{S} 4 \mathrm{~A} \uparrow$. The thin constituent, petal-like component nanosheets measured $12.5 \pm 2.6 \mathrm{~nm}$ in thickness from the TEM image, as shown in Figure S4B $\uparrow$. The morphologies and micronscale structures of the LTO-MWNT ( $5 \mathrm{wt} \%$ ) composites were investigated by TEM and SEM, as presented in Figure 2. The MWNTs, indicated by arrows, measure 10-30 nm in diameter and are intermingled with the LTO spheres.

The composite generated through a physical sonication method (Figure 2A and B) evinced the presence of a relatively uniform coverage of evenly distributed MWNTs with no observable morphological change associated with the underlying LTO micron-scale spheres. As for the corresponding composite synthesized by the direct in situ deposition technique, the presence of the MWNTs might have potentially interfered with the growth of the LTO micron-scale spheres themselves during the process of the hydrothermal reaction, since we observed a certain degree of fragmentation, thereby resulting in the formation of 'broken up', particulate LTO structures, possessing an average diameter of $35.2 \pm 5.3 \mathrm{~nm}$, as well as individual, dissociated nanosheets, ${ }^{1}$ as indicated by the white circles in Figures $2 \mathrm{C}$ and D.

By contrast, we noted that the MWNTs appeared to be more uniformly dispersed and distributed throughout the network of LTO micron-scale spheres within the framework of composites generated through a covalent attachment strategy (Figures $2 \mathrm{E}$ and F). However, the occurrence of individual nanosheets themselves became less 
distinctive, possibly due to the presence of surface capping associated with APTES linker molecules, as indicated by the yellow circles in Figure 2F. By analogy with the LTOMWNT composite derived from physical sonication, the composite prepared by noncovalent $\pi-\pi$ interactions (Figure $2 \mathrm{G}$ and $\mathrm{H}$ ) also evinced a uniform MWNT coverage on the surface with little if any noticeable morphological alteration of the LTO motif.

More detailed structural information was provided by HRTEM images and selected area electron diffraction (SAED) patterns (Figure 3A-D). In effect, within the HRTEM data, distinctive lattice fringes possessing distances of approximately $4.84 \AA$ and $3.41 \AA$ were observed, corresponding to the (111) planes of spinel LTO and the interlayer spacings of graphitic layers within the MWNTs themselves, respectively. The corresponding SAED patterns could be indexed to the (111), (311), and (400) lattice planes of spinel LTO as well as to the (002) lattice plane of MWNTs, respectively.

These data provided further evidence for the presence of phase purity within our system. We found that the MWNTs are in close contact with the LTO nanosheets, thereby providing for the possibility of enhanced electron transport pathways between adjacent LTO micron-scale spheres. It is also noteworthy that the covalently attached composite (Figure 3C inset) was less crystalline than for the other three as-formed heterostructures (Figure 3A, B, and D inset), an observation which again was consistent with the aforementioned XRD results.

In order to visualize and further reveal structural information at the junctions between the MWNTs and the LTO microspheres, EDS mapping data of the composites variously prepared by the four different attachment modalities studied herein are provided in Figures S5-8 $\uparrow$ of the Supporting Information. It should be noted that the C 
mapping signals are relatively diffuse in all four samples, due to the presence of a carbon supporting film on the Cu TEM grid. Nonetheless, it is expected that MWNTs and LTO microspheres are in direct contact with each other in samples prepared by both sonication (Figure $\mathrm{S} 5 \dagger$ ) and in situ deposition (Figure S6†) methods, and not surprisingly, only $\mathrm{Ti}$, $\mathrm{O}$, and $\mathrm{C}$ signals can be detected. By contrast, in the sample prepared by the covalent attachment strategy (Figure S7 $\dagger$ ), additional $\mathrm{N}$ and Si signals likely stemming from the APTES linker were observed. These signals spatially overlap rather well with the corresponding $\mathrm{Ti}$ and $\mathrm{O}$ peaks associated with the LTO microspheres, thereby implying a relatively uniform coating of APTES molecules onto the underlying LTO surface.

Similarly, mapping results of the S element emanating from the 4-MBA linker coincide closely with those of $\mathrm{Ti}$ and $\mathrm{O}$ within the sample prepared by $\pi-\pi$ interactions (Figure S8†). These data likewise unambiguously confirm the expected presence of 4-MBA molecules at the junctions between the LTO and the MWNT, a finding consistent with the sample preparation conditions.

FT-IR spectroscopy was used to confirm the formation of amide bonds within the LTO-MWNT composite formed by means of covalent attachment. Specifically, spectra of (i) oxidized MWNTs, (ii) pure LTO, (iii) APTES, as well as (iv) 4-MBAfunctionalized LTO, together with (v) LTO - MWNT $5 \mathrm{wt} \%$ composites, generated by both covalent attachment and $\pi-\pi$ interaction methods, are respectively shown in Figure 4a-f. Upon treatment with nitric acid, the peaks located at $1720 \mathrm{~cm}^{-1}$ (Figure 4a) could be ascribed to the stretching bands of the $\mathrm{C}=\mathrm{O}$ functionality derived from the carboxylic acid group, ${ }^{40}$ thereby confirming the success of the acid functionalization protocol. It was noted that no distinctive peaks appeared for the pure LTO sample (Figure 4b). In the 
APTES-LTO species (Figure 4c), the characteristic peaks located at 1140 and $1030 \mathrm{~cm}^{-1}$

could be assigned to the Si-O-H and $\mathrm{Si}-\mathrm{O}-\mathrm{Si}$ groups associated with the APTES linker, ${ }^{41}$ whereas the peaks localized at $3430 \mathrm{~cm}^{-1}$ and $1650 \mathrm{~cm}^{-1}$ were consistent with the N-H stretching and bending modes derived from the amine group, respectively. These data were indicative of the likely successful functionalization involving APTES.

Peaks situated at $1650 \mathrm{~cm}^{-1}, 3406 \mathrm{~cm}^{-1}$, and $1577 \mathrm{~cm}^{-1}$ and associated within the LTO-MWNT composite derived from the covalent attachment method (Figure 4e) likely corresponded to the $\mathrm{C}=\mathrm{O}$ stretching bands in addition to the $\mathrm{N}-\mathrm{H}$ stretching and bending modes from the amide group, respectively, all of which were suggestive of the probable formation of an amide bond between the MWNTs and the LTO micron-scale spheres. The 4-MBA coated LTO sample (Figure 4d) gave rise to a sharp peak located at $1680 \mathrm{~cm}^{-}$ ${ }^{1}$, corresponding to the stretching mode of the $\mathrm{C}=\mathrm{O}$ bond associated with the 4-MBA linker. Peaks located at 1591 and $1425 \mathrm{~cm}^{-1}$ could be ascribed to the stretching mode of the phenyl ring derived from the linker, an observation consistent with a successful functionalization process. After attaching the MBA-coated LTO onto the MWNTs (Figure 4f), the ring stretching peak shifted from $1425 \mathrm{~cm}^{-1}$ to $1398 \mathrm{~cm}^{-1}$, a result which was likely induced mainly by strong $\pi-\pi$ stacking interactions between the phenyl ring in the MBA linker and the conjugated MWNT network, and consequently, a 'softening' of the $\mathrm{C}=\mathrm{C}$ bonds, i.e. indicative of an expansion of $\mathrm{C}-\mathrm{C}$ bonds. ${ }^{42,43}$

\subsection{Electrochemical properties of LTO-MWNT composite heterostructures}

\subsubsection{Cyclic voltammetry}


Cyclic voltammetry of two electrode cells containing the LTO-MWNT heterostructures was collected at scan rates of $0.5,1.0,2.0$, and $5.0 \mathrm{mV} / \mathrm{s}$, as shown in Figure 5. The voltammograms of the $5 \%$ in situ sample revealed reversible electrochemistry at all scan rates wherein clear anodic and cathodic peaks were apparent, as indicated in Figure 5a. The $\Delta \mathrm{E}_{\text {peak }}$ values were $0.36,0.36,0.48$, and $0.60 \mathrm{~V}$ at the scan rates of $0.5,1.0,2.0$, and $5.0 \mathrm{mV} / \mathrm{s}$, respectively. Under the slower scan rates of 0.5 and $1.0 \mathrm{mV} / \mathrm{sec}$, two features were present for the anodic wave. In data associated with the 'in situ' prepared sample, the reduction peak noted at $1.4 \mathrm{~V}$ can be attributed to the irreversible formation of the $\mathrm{LiTiO}_{2}$ phase from the rutile $\mathrm{TiO}_{2}$ phase, ${ }^{44,45}$ as determined from refinement of the relevant $\mathrm{XRD}$ profile, as illustrated in Figure $\mathrm{S} 3 \mathrm{C} \uparrow$. At the highest scan rate of $5.0 \mathrm{mV} / \mathrm{s}$, the cathodic wave showed distortion, leading to a broadening of the appearance of the peak. The 5\% physically sonicated LTO-MWNT sample demonstrated $\Delta \mathrm{E}_{\text {peak }}$ values of $0.36,0.44,0.56$, and $0.70 \mathrm{~V}$ at scan rates of $0.5,1.0,2.0$, and $5.0 \mathrm{mV} / \mathrm{s}$, respectively, as illustrated in Figure $5 \mathrm{~b}$. The cathodic wave at the 5.0 $\mathrm{mV} / \mathrm{sec}$ scan rate also gave rise to distortion, thereby leading to a broadening of the appearance of the peak.

The CV for the $5 \%$ covalent LTO-MWNT sample (Figure $5 \mathrm{c}$ ) showed $\Delta \mathrm{E}_{\text {peak }}$ values which were $0.32,0.44,0.62$, and $0.93 \mathrm{~V}$ at scan rates of $0.5,1.0,2.0$, and $5.0 \mathrm{mV} / \mathrm{s}$, respectively. The $\Delta \mathrm{E}_{\text {peak }}$ values for the covalently attached sample were comparable to the in situ and the sonicated samples measured at scan rates of 0.5 to $2.0 \mathrm{mV} / \mathrm{s}$, yet these were higher at the scan rate of $5.0 \mathrm{mV} / \mathrm{s}$. It is noteworthy that the $\mathrm{CV}$ peaks associated with the covalently attached composites are wider as compared with the other three preparative analogues, an observation which can be ascribed to the larger charge transfer 
resistance imparted by the presence of the APTES linkers, as demonstrated in the EIS data analyzed in the following discussion.

By comparison, the LTO-MWNT composite prepared through non-covalent $\pi-\pi$ interactions displayed $\Delta \mathrm{E}_{\text {peak }}$ values of $0.25,0.35,0.45$, and $0.61 \mathrm{~V}$ at scan rates of 0.5 , 1.0, 2.0, and $5.0 \mathrm{mV} / \mathrm{s}$, respectively, as shown in Figure $5 \mathrm{~d}$. The smallest $\Delta \mathrm{E}_{\text {peak }}$ values among all samples analyzed at each scan rate and therefore, the fastest kinetic behavior observed are noted with this LTO-MWNT composite, prepared with the MBA linker. It is also worth highlighting that this sample exhibited the best consistency among all of the variously prepared LTO-MWNT composites tested.

For all of the prepared samples, the anodic and cathodic peak current values were acquired and plotted versus the square root of the scan rate, as shown in Figure 6, in order to determine (i) if the charge transfer kinetics were fast enough to obey the RandlesSevcik equation, ${ }^{46}$ and equally significantly, (ii) the Li-ion diffusion coefficients of all of the samples analyzed. The sample prepared by the physical sonication method yielded correlation coefficients of 0.99 and 0.96 for the cathodic and anodic peak currents, respectively. The in situ sample gave rise to correlation coefficients of 0.99 and 0.98 for the cathodic and anodic peak currents, while the covalent sample highlighted correlation coefficients of 0.99 and 0.96 for the cathodic and anodic peak currents, respectively. The sample prepared through $\pi-\pi$ interactions generated coefficients of 0.99 for both the cathodic and anodic peak currents. These results indicate that the fastest kinetics among the four attachment modalities were exhibited by the LTO-MWNT sample generated using non-covalent $\pi-\pi$ interactions, a composite whose formation was mediated by the aromatic, conjugated 4-MBA linker. 
Based upon the Randles-Sevcik equation shown below, the Li-ion diffusion coefficient can be calculated from the slope of the graph, obtained by plotting $i_{p}$ as a function of the square root of the scan rate.

$$
t_{p}=2.69 * 10^{5} n^{\frac{8}{2}} A D_{0}^{\frac{1}{2}} C_{0}^{*} v^{1 / 2}
$$

The Li-ion diffusion coefficients are found to be $3.4 \times 10^{-8}, 1.5 \times 10^{-7}, 5.9 \times 10^{-8}$,

and $2.3 \times 10^{-7} \mathrm{~cm}^{2} / \mathrm{s}$, for the samples derived from physical sonication, in-situ deposition, covalent attachment, and non-covalent $\pi-\pi$ interactions, respectively. The sample prepared by non-covalent $\pi-\pi$ interactions yielded a much higher diffusion coefficient as compared with the other sample types. Therefore, we can conclude that the diffusion coefficient and charge transfer are likely to be favorable for the $\pi-\pi$ interaction sample.

\subsubsection{Galvanostatic charge-discharge cycling and rate capability}

The electrochemical cells were tested in galvanostatic mode for both discharge and charge processes. The LTO-MWNT composite samples were subjected to a total of 105 discharge-charge cycles, consisting of three rounds of 35 cycles at discharge rates of C/2 (20 cycles), $20 \mathrm{C}$ (5 cycles), $50 \mathrm{C}$ (5 cycles), and $100 \mathrm{C}$ (5 cycles). This testing provided the opportunity to assess both rate capability and capacity retention. Different strategies of integration with and attachment of MWNTs onto LTO were investigated on samples possessing 5\% MWNT loading amounts, as exemplified in Figure 7.

The capacity retention for the four as-generated LTO-MWNT composites was investigated. At cycles 20,55 , and 90 , the capacities for the composite generated by the covalent method using the APTES linker were measured to be 148,145 , and $143 \mathrm{mAh} / \mathrm{g}$, respectively, under a rate of $\mathrm{C} / 2$. The capacities for the composite synthesized by the in 
situ protocol were found to be 150,150 , and $149 \mathrm{mAh} / \mathrm{g}$, while the capacities for the composite derived from the physical sonication technique were computed to be 161,160 , and $159 \mathrm{mAh} / \mathrm{g}$, respectively. Notably, the capacities for the non-covalent sample generated with the MBA linker were the highest of the samples tested; specifically, the data yielded values of 174,171 , and $170 \mathrm{mAh} / \mathrm{g}$. Thus, the LTO-MWNT sample prepared using the covalent method exhibited a slightly poorer capacity retention of $\sim 97 \%$ from cycles $20-90$ as compared with the samples prepared via either physical sonication, in situ direct deposition, or $\pi-\pi$ interaction methods, all of which yielded $\sim 99 \%$ capacity retention from cycles $20-90$.

At a rate of $\mathrm{C} / 2$, whereas the sample prepared by physical sonication yielded a capacity of $161 \mathrm{mAh} \mathrm{g}^{-1}$, the analogous LTO-MWNT $5 \mathrm{wt} \%$ composite fabricated by the $\pi-\pi$ interaction protocol out-performed all of the other materials tested. In fact, this noncovalently derived composite delivered a capacity of $175 \mathrm{mAh} \mathrm{g}^{-1}$, essentially achieving the expected theoretical capacity predicted for the LTO material. The differences among the measured capacities under the $\mathrm{C} / 2$ rate for the LTO-MWNT $5 \mathrm{wt} \%$ composites, synthesized by the in situ direct-deposition approach as well as by covalent attachment protocols were minimal, yielding 150 and $148 \mathrm{mAh} / \mathrm{g}$, respectively.

The rate capability of the material samples was also assessed, as revealed in Figure 7. Under C/2 conditions, both the in situ prepared LTO-MWNT sample and the sample prepared via physical sonication demonstrated an abrupt voltage drop upon initial discharge to $\sim 20 \mathrm{mAh} / \mathrm{g}$, followed by a broad voltage plateau at $\sim 1.55 \mathrm{~V}$, as indicated in Figures $7 \mathrm{a}$ and $7 \mathrm{~b}$, respectively. The sample generated by the non-covalent $\pi-\pi$ interaction utilizing the MBA linker displayed a slightly more gradual voltage drop upon 
initial discharge, followed by the appearance of a long plateau at $\sim 1.55 \mathrm{~V}$ (Figure $7 \mathrm{~d}$ ).

The sample prepared using the covalent attachment with the APTES linker gave rise to a more gradually sloping voltage profile with higher voltages from $2.2-1.6 \mathrm{~V}$ out to 60 $\mathrm{mAh} / \mathrm{g}$, and followed by a plateau at $\sim 1.55 \mathrm{~V}$ for the remainder of the discharge process, as shown in Figure 7c. Even though the realized capacities for the four electrode types were similar under low rate discharge conditions, dissimilarities in the shapes of the voltage profiles became rather more apparent and pronounced at higher rates.

Under high rates of discharge, more significant differences were observed, wherein the highest delivered capacity was achieved by the samples prepared via the $\pi-\pi$ interaction method. For example, under a $50 \mathrm{C}$ discharge rate, these latter non-covalently derived samples delivered $145 \mathrm{mAh} / \mathrm{g}$, whereas the sonication-induced, in situ-derived, as well as covalently-generated samples only produced 90,71 , and $16 \mathrm{mAh} / \mathrm{g}$, respectively. Notably, the covalently derived samples with the APTES linker had no functional capacity under a higher $100 \mathrm{C}$ discharge rate, whereas the samples prepared by physical sonication, the in situ deposition method, and the non-covalent $\pi-\pi$ interaction protocols produced capacities of 15,8 , and $77 \mathrm{mAh} / \mathrm{g}$ at a similar $100 \mathrm{C}$ discharge rate. Significantly, the sample derived from the $\pi-\pi$ interaction protocol using the 4-MBA aromatic, conjugated linker gave rise to a capacity of $77 \mathrm{mAh} / \mathrm{g}$ with an extended plateau at $\sim 1.16 \mathrm{~V}$, thereby rendering it as the most promising high-rate LTO heterostructure.

Under cycling, the samples prepared via the physical sonication method delivered $161,145,90$, and $15 \mathrm{mAh} / \mathrm{g}$, under discharge rates of $\mathrm{C} / 2,20 \mathrm{C}, 50 \mathrm{C}$, and $100 \mathrm{C}$, at cycles 20, 25, 30, and 35, respectively, as demonstrated in Figure 8. By contrast, the 5\% in situ LTO-MWNT material resulted in capacities of $150,133,71$, and $8 \mathrm{mAh} / \mathrm{g}$, while 
the sample prepared by the covalent method using the APTES linker gave rise to corresponding capacities of $148,105,16$, and $0.15 \mathrm{mAh} / \mathrm{g}$, respectively, through cycles $20,25,30$, and 35 . It is worth noting that the 'control' sample, i.e. the pure 3D flower LTO without any MWNT incorporation, yielded capacity values of 153, 141, 109, and 24 $\mathrm{mAh} / \mathrm{g}$, respectively, through cycles $20,25,30$, and 35 .

To interpret these data, we highlight that the sample prepared by physical sonication method showed improvement at $\mathrm{C} / 2$ and $20 \mathrm{C}$ discharge rates, but the samples derived from both in situ and covalent attachment strategies displayed lower capacity readings as compared with the pure LTO. By contrast, the sample prepared from $\pi-\pi$ interactions using the aromatic, conjugated 4-MBA linker displayed outstanding performance by giving rise to capacity values of $174,163,146$ and $77 \mathrm{mAh} / \mathrm{g}$ for cycles $20,25,30$, and 35 respectively. By means of comparison with the 'control' sample itself, the sample with the aromatic, conjugated MBA linker delivered $37 \mathrm{mAh} / \mathrm{g}$ higher capacity than the pure, unmodified 3D flower LTO sample at a $50 \mathrm{C}$ discharge rate and $53 \mathrm{mAh} / \mathrm{g}$ higher capacity at a $100 \mathrm{C}$ discharge rate.

In all cases, the trend in the charge capacities reflected the corresponding changes in the discharge capacities measured under the same rate. These quantifiable differences are consistent with prior observations from the CV experiments. Indeed, (i) the high reversible capacity and the excellent cycling stability at $\mathrm{C} / 2$ coupled with (ii) the high discharge rates of $20 \mathrm{C}$ and $50 \mathrm{C}$ for the LTO-MWNT $5 \mathrm{wt} \%$ composites, generated by non-covalent $\pi-\pi$ interactions using the aromatic, conjugated 4-MBA linker, are clearly superior to those of previously reported values associated with LTO-carbon nanotube 
composite motifs. A detailed comparison of our results in the context of the existing literature is presented in Table S1 (See ESI).

\subsubsection{Electrochemical impedance spectroscopy (EIS) data}

Electrochemical impedance spectroscopy was used to compare the inherent resistance of the Li/LTO-MWNT cells with 5\% MWNT loading, prepared using different modes of attachment, as pointed out in Figure 9. The data were fit using an equivalent circuit, where R1 was attributed to ohmic resistance and R2 was ascribed to charge transfer resistance. The $\mathrm{R} 1$ values were consistent for all four cell types, with a range of $1.5-2.3$ ohms. The R2 quantity, however, gave rise to more variation among the cells, with measured values of 52, 65, and 89 ohms noted for the cells prepared from LTOMWNT samples associated with sonication, in situ deposition, and covalent attachment modalities, respectively.

The higher charge transfer resistance ascribed to the covalent attachment modality in particular was consistent with the poorer rate capability discussed above in the context of the voltammetry and galvanostatic testing results. Moreover, these results are consistent with a study, wherein covalent attachment of $\mathrm{Fe}_{3} \mathrm{O}_{4}$ onto a glassy carbon electrode surface using a 3-aminopropyltriethoxysilane (APTES) linker led to the observation of a higher charge transfer resistance as compared with a bare glassy carbon surface. ${ }^{47}$ To account for this behavior, we note that it has been reported that the presence of unwieldy, sterically bulky, and non-conjugated ligands can functionally act as an undesirable potential barrier which can thereby inhibit the degree of charge transport between adjacent nanoparticles and nanostructures. ${ }^{48,49}$ An increased charge transfer resistance associated with the covalent attachment process using APTES would also 
account for not only the lower delivered capacity but also, due to increased polarization effects, the poorer capacity retention observed.

By contrast, the sample generated from the non-covalent $\pi-\pi$ interaction method using the aromatic, conjugated 4-MBA linker displayed only 24 ohms as an R2 value, indicating the most favorable charge transfer process occurring among all of the LTOMWNT composites tested. This finding moreover corroborates previous published results which suggest that electron-rich, conjugated systems are more efficacious at enabling the charge transfer process as compared with their non-conjugated counterparts. ${ }^{48}$

\section{Conclusions}

In the current manuscript, we have demonstrated the successful synthesis, for the first time, of "flower-like" LTO-MWNT composites systematically fabricated via different preparative approaches, including (i) physical sonication, (ii) an in situ direct deposition approach, (iii) a covalent chemical attachment protocol, as well as (iv) a $\pi-\pi$ interaction strategy. Data from our structural characterization analysis suggest that the composites generated by both the physical sonication and non-covalent $\pi-\pi$ interaction methods retain not only the favorably small crystallite size (i.e. $12.6 \mathrm{~nm}$ ) of the pure LTO but also the intrinsic "flower-like" morphology ascribed to pure LTO, both during and after the preparative process.

By contrast, the composite prepared through an in situ direct-deposition approach yielded not only fragmented LTO structures possibly due to curtailed crystal growth induced by the presence of interfering MWNTs but also larger overall crystallite sizes likely resulting from the higher annealing temperatures used, denoting factors which 
contributed to the lower electrochemical performance measured. Rietveld refinement results also suggested the existence of the rutile and anatase $\mathrm{TiO}_{2}$ impurities within the composite, which were detected in the CV peaks. Finally, the composite produced via covalent attachment appeared to be enveloped with a coating of amorphous linker molecules, thereby leading to not only lower overall crystallinity but also a decreased contact area between LTO and MWNTs, thereby giving rise to poorer performance.

Equally significant is that we have correlated the electrochemical performance of these composite materials with their corresponding attachment chemistry. For example, in this study, the LTO sample with the 5\% MWNT loading prepared via the $\pi$ - $\pi$ noncovalent interaction method evidenced the highest delivered discharge capacity at every $\mathrm{C}$ rate from $\mathrm{C} / 2$ to $100 \mathrm{C}$ with the most notable differences apparent under discharge rates $\geq 20 \mathrm{C}$, due to a much lower charge transfer resistance as compared with those of the other LTO-MWNT composites analyzed. It is worth further mentioning that these LTOMWNT composites, produced by means of $\pi-\pi$ non-covalent interactions, exhibited a reproducibly high rate capability and a desirable cycling stability, i.e. delivering $174 \mathrm{~mA}$ $\mathrm{h} \mathrm{g}^{-1}$ at $\mathrm{C} / 2$ with a $99 \%$ capacity retention from cycles $20-90,163 \mathrm{~mA} \mathrm{~h} \mathrm{~g}^{-1}$ at $20 \mathrm{C}$ with a $97 \%$ capacity retention from cycles $25-95$, and $146 \mathrm{mAh} / \mathrm{g}$ at $50 \mathrm{C}$ with a $90 \%$ capacity retention from cycles 30-100.

These values denote, to the best of our knowledge, clearly superior performance to those of any previously reported LTO-carbon nanotube composite materials, to date, especially under these relatively low loading conditions. Notably, the LTO-MWNT samples prepared via the covalent attachment scheme delivered a lower capacity and displayed $97 \%$ capacity retention from cycle 20 to cycle 90 at $\mathrm{C} / 2$ rate as compared with 
the higher capacity and $99 \%$ capacity retention for the set of physically sonicated, in situ, and $\pi-\pi$ interaction samples. The voltammetric and galvanostatic data coupled with the impedance results indicate slower kinetics for the LTO-MWNT heterostructures, prepared using the covalent attachment approach, denoting data consistent with a prior report on a totally different system wherein increased charge transfer resistance was found to have been associated with a covalent coupling protocol involving the 3 aminopropyltriethoxysilane (APTES) linker. ${ }^{47}$

Hence, our work illustrates a clear example, wherein we have used deliberative processing protocols in order to tune and control fundamental material properties. Indeed, such a truly favorable cycling performance coupled with the high discharge capacity detected highlight distinctive advantages for our hierarchical architectures from a battery perspective. In addition, together with the rather simplistic attachment route used and the fairly economical production costs associated with fabricating our "flower-like" LTOMWNT $5 \mathrm{wt} \%$ composites generated by the $\pi-\pi$ interaction method using the aromatic, conjugated 4-MBA linker, these cumulative results suggest the practical viability of utilizing these novel hierarchical structures within the context of commercially relevant designs for energy storage.

\section{Acknowledgements}

All of the work described in these studies was funded as part of the Center for Mesoscale Transport Properties (m2M), an Energy Frontier Research Center supported by the U.S. Department of Energy, Office of Science, Basic Energy Sciences, under award \#DE-SC0012673. Research characterization was carried out in part at the Center 
for Functional Nanomaterials, Brookhaven National Laboratory, which is supported by the U.S. Department of Energy, Office of Basic Energy Sciences, under Contract No. DE-SC0012704. We thank Dr. Chung-Chueh Chang for assistance with EDS mapping data acquisition, associated with the MWNT-LTO composite samples. 


\section{References}

1. C. F. Lin, X. Y. Fan, Y. L. Xin, F. Q. Cheng, M. O. Lai, H. H. Zhou and L. Lu, J. Mater. Chem. A, 2, 9982 (2014).

2. J. Guo, W. Zuo, Y. Cai, S. Chen, S. Zhang and J. Liu, J. Mater. Chem. A, 3, 4938 (2015).

3. Y. Sha, B. Zhao, R. Ran, R. Cai and Z. Shao, J. Mater. Chem. A, 1, 13233 (2013).

4. G. N. Zhu, Y. G. Wang and Y. Y. Xia, Energy Environ. Sci., 5, 6652 (2012).

5. Y. F. Tang, L. Yang, S. H. Fang and Z. Qiu, Electrochim. Acta, 54, 6244 (2009).

6. J. L. Qiao, Y. Y. Liu, F. Hong and J. J. Zhang, Chem. Soc. Rev., 43, 631 (2014).

7. J.-H. Choi, W.-H. Ryu, K. Park, J.-D. Jo, S.-M. Jo, D.-S. Lim and I.-D. Kim, Sci. Rep., 4, 7334 (2014).

8. $\quad$ S. Kim, S. H. Fang, Z. X. Zhang, J. Z. Chen, L. Yang, J. E. Penner-Hahn and A. Deb, J. Power Sources, 268, 294 (2014).

9. $\quad$ H. F. Ni and L. Z. Fan, J. Power Sources, 214, 195 (2012).

10. J. Lim, E. Choi, V. Mathew, D. Kim, D. Ahn, J. Gim, S. H. Kang and J. Kim, J. Electrochem. Soc., 158, A275 (2011).

11. L. Sun, J. P. Wang, K. L. Jiang and S. S. Fan, J. Power Sources, 248, 265 (2014).

12. L. F. Shen, E. Uchaker, X. G. Zhang and G. Z. Cao, Adv. Mater., 24, 6502 (2012).

13. J. Liu, K. P. Song, P. A. van Aken, J. Maier and Y. Yu, Nano Lett., 14, 2597 (2014).

14. J. Z. Chen, L. Yang, S. H. Fang and Y. F. Tang, Electrochim. Acta, 55, 6596 (2010).

15. D. Kong, W. Ren, Y. Luo, Y. Yang and C. Cheng, J. Mater. Chem. A, 2, 20221 (2014).

16. H. Xia, Z. T. Luo and J. P. Xie, Nanotechnol. Rev., 3, 161 (2014).

17. L. Wang, Y. M. Zhang, M. E. Scofield, S. Y. Yue, C. McBean, A. C. Marschilok, K. J. Takeuchi, E. S. Takeuchi and S. S. Wong, ChemSusChem, 8, 3304 (2015).

18. L. Shen, X. Zhang, E. Uchaker, C. Yuan and G. Cao, Adv. Energy Mater., 2, 691 (2012).

19. G. B. Xu, W. Li, L. W. Yang, X. L. Wei, J. W. Ding, J. X. Zhong and P. K. Chu, J. Power Sources, 276, 247 (2015).

20. W. D. Zhang, B. Xu and L. C. Jiang, J. Mater. Chem., 20, 6383 (2010).

21. A. Marschilok, C. Y. Lee, A. Subramanian, K. J. Takeuchi and E. S. Takeuchi, Energy Environ. Sci., 4, 2943 (2011).

22. J. J. Huang and Z. Y. Jiang, Electrochim. Acta, 53, 7756 (2008).

23. Y. R. Jhan and J. G. Duh, J. Power Sources, 198, 294 (2012).

24. X. L. Jia, Y. F. Kan, X. Zhu, G. Q. Ning, Y. F. Lu and F. Wei, Nano Energy, 10, 344 (2014).

25. H. K. Kim, K. C. Roh, K. Kang and K. B. Kim, RSC Adv., 3, 14267 (2013).

26. J. Shu, L. Hou, R. Ma, M. Shui, L. Y. Shao, D. J. Wang, Y. L. Ren and W. D.

Zheng, RSC Adv., 2, 10306 (2012). 
27. P. Zhang, M. Chen, X. Shen, Q. Wu, X. Zhang, L. Huan and G. Diao, Electrochim. Acta, 204, 92 (2016).

28. W. Fang, P. J. Zuo, Y. L. Ma, X. Q. Cheng, L. X. Liao and G. P. Yin, Electrochim. Acta, 94, 294 (2013).

29. L. F. Shen, C. Z. Yuan, H. J. Luo, X. G. Zhang, K. Xu and F. Zhang, J. Mater. Chem., 21, 761 (2011).

30. N. Li, T. Mei, Y. Zhu, L. Wang, J. Liang, X. Zhang, Y. Qian and K. Tang, CrystEngComm, 14, 6435 (2012).

31. Y. Tang, F. Huang, W. Zhao, Z. Liu and D. Wan, J. Mater. Chem., 22, 11257 (2012).

32. S. Sankaraiah, J. M. Lee, J. H. Kim and S. W. Choi, Macromolecules, 41, 6195 (2008).

33. I. Noda, T. Kamoto and M. Yamada, Chem. Mater., 12, 1708 (2000).

34. E. Asenath-Smith and W. Chen, Langmuir, 24, 12405 (2008).

35. A. Raman, R. Quinones, L. Barriger, R. Eastman, A. Parsi and E. S. Gawalt, Langmuir, 26, 1747 (2010).

36. H.-K. Kim, K. C. Roh, K. Kang and K.-B. Kim, RSC Adv., 3, 14267 (2013).

37. G. Hasegawa, K. Kanamori, T. Kiyomura, H. Kurata, K. Nakanishi and T. Abe, Adv. Energy Mater., 5, 1400730 (2015).

38. Y. B. Shen, J. R. Eltzholtz and B. B. Iversen, Chem. Mater., 25, 5023 (2013).

39. H.-Y. Cheng, L.-J. Lai and F.-H. Ko, Int. J. Nanomedicine, 7, 2967 (2012).

40. Y. Si and E. T. Samulski, Nano Lett., 8, 1679 (2008).

41. R. Villalonga, M. L. Villalonga, P. Diez and J. M. Pingarron, J. Mater. Chem., 21, 12858 (2011).

42. D.-Q. Yang, J.-F. Rochette and E. Sacher, J. Phys. Chem. B, 109, 4481 (2005).

43. Y. Zhang, S. Yuan, W. Zhou, J. Xu and Y. Li, J. Nanosci. Nanotechnol., 7, 2366 (2007).

44. D. Wang, D. Choi, Z. Yang, V. V. Viswanathan, Z. Nie, C. Wang, Y. Song, J.-G. Zhang and J. Liu, Chem. Mater., 20, 3435 (2008).

45. Y. Bai, Z. M. Liu, N. Q. Zhang and K. N. Sun, RSC Adv., 5, 21285 (2015).

46. A. J. Bard and L. R. Faulkner, Electrochemical Methods: Fundamentals and Applications, Wiley, New York (2000).

47. H. S. Yin, Y. L. Zhou, T. Liu, T. T. Tang, S. Y. Ai and L. S. Zhu, J. Solid State Electrochem., 16, 731 (2012).

48. L. Wang, J. Han, B. Sundahl, S. Thornton, Y. Zhu, R. Zhou, C. Jaye, H. Liu, Z. Q. Li, G. T. Taylor, D. A. Fischer, J. Appenzeller, R. J. Harrison and S. S. Wong, Nanoscale, 8, 15553 (2016).

49. T. Virgili, I. S. López, B. Vercelli, G. Angella, G. Zotti, J. Cabanillas-Gonzalez, D. Granados, L. Luer, R. Wannemacher and F. Tassone, J. Phys. Chem. C, 116, 16259 (2012). 


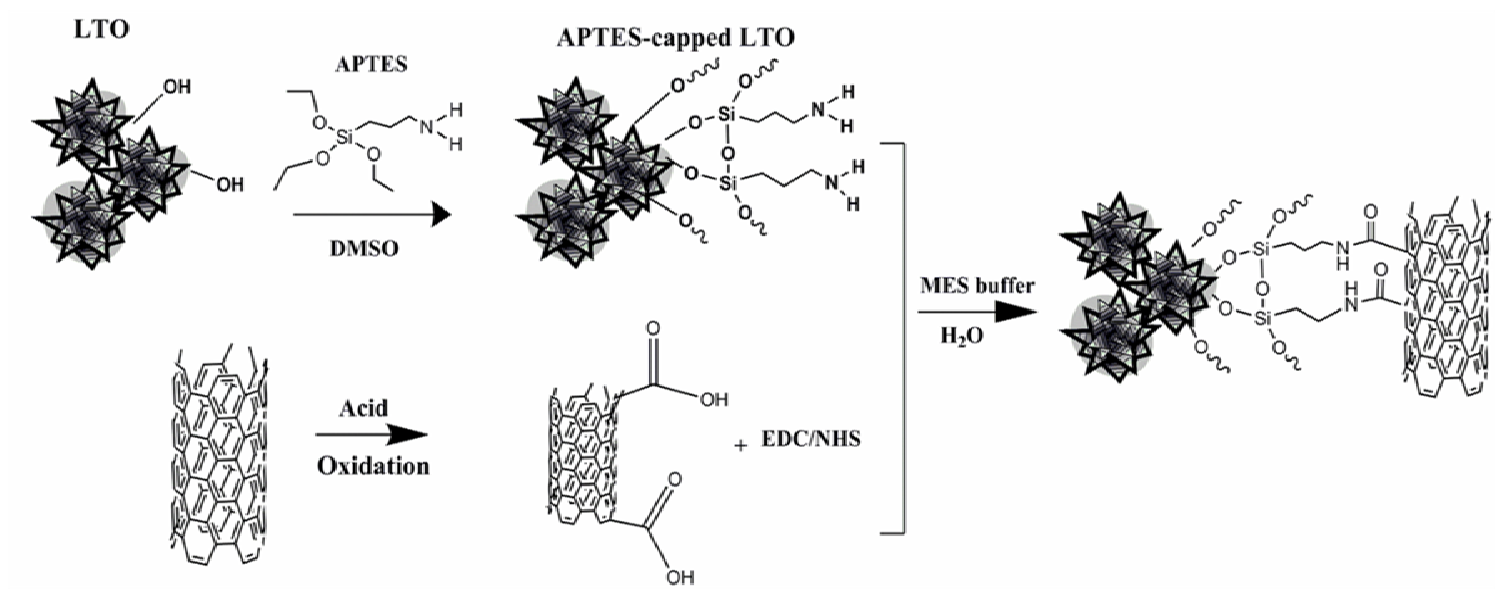

Scheme 1. Reaction schematic denoting the covalent attachment process between 'flower-shape' LTO micron-scale spheres and the adjoining MWNTs. 

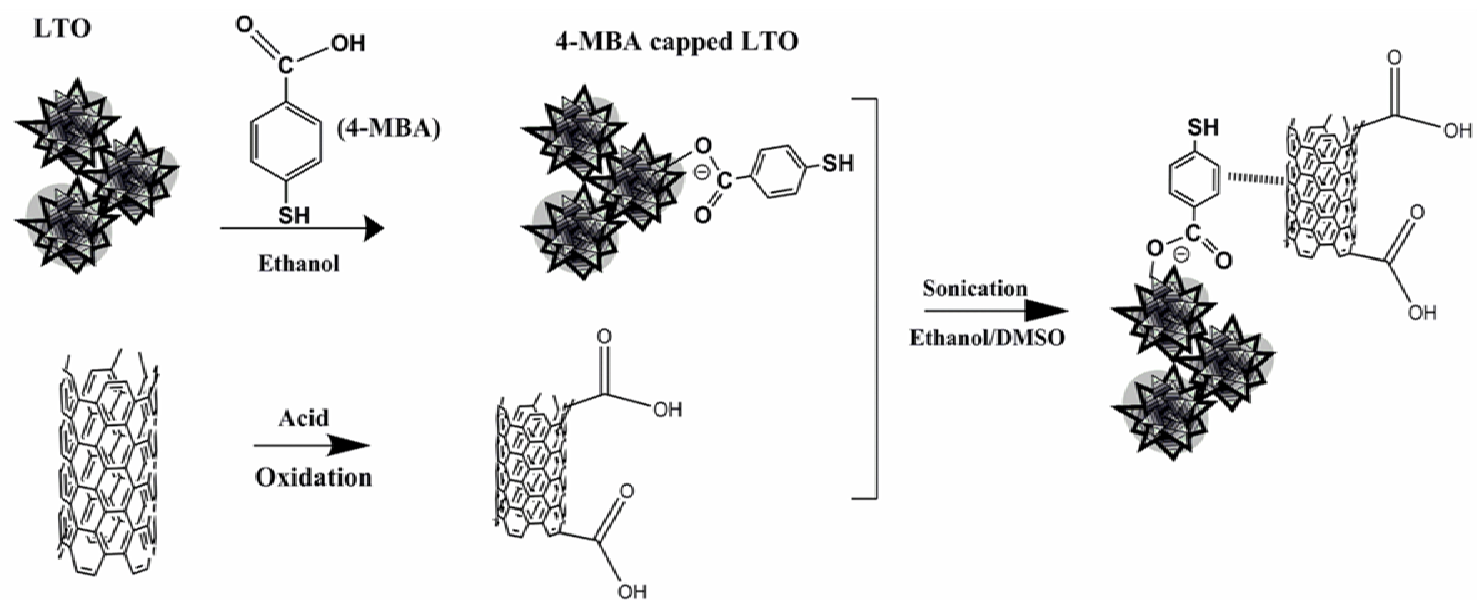

Scheme 2. Reaction schematic demonstrating the non-covalent $\pi-\pi$ interactions between the 'flower-shape' LTO micron-scale spheres and MWNTs. 


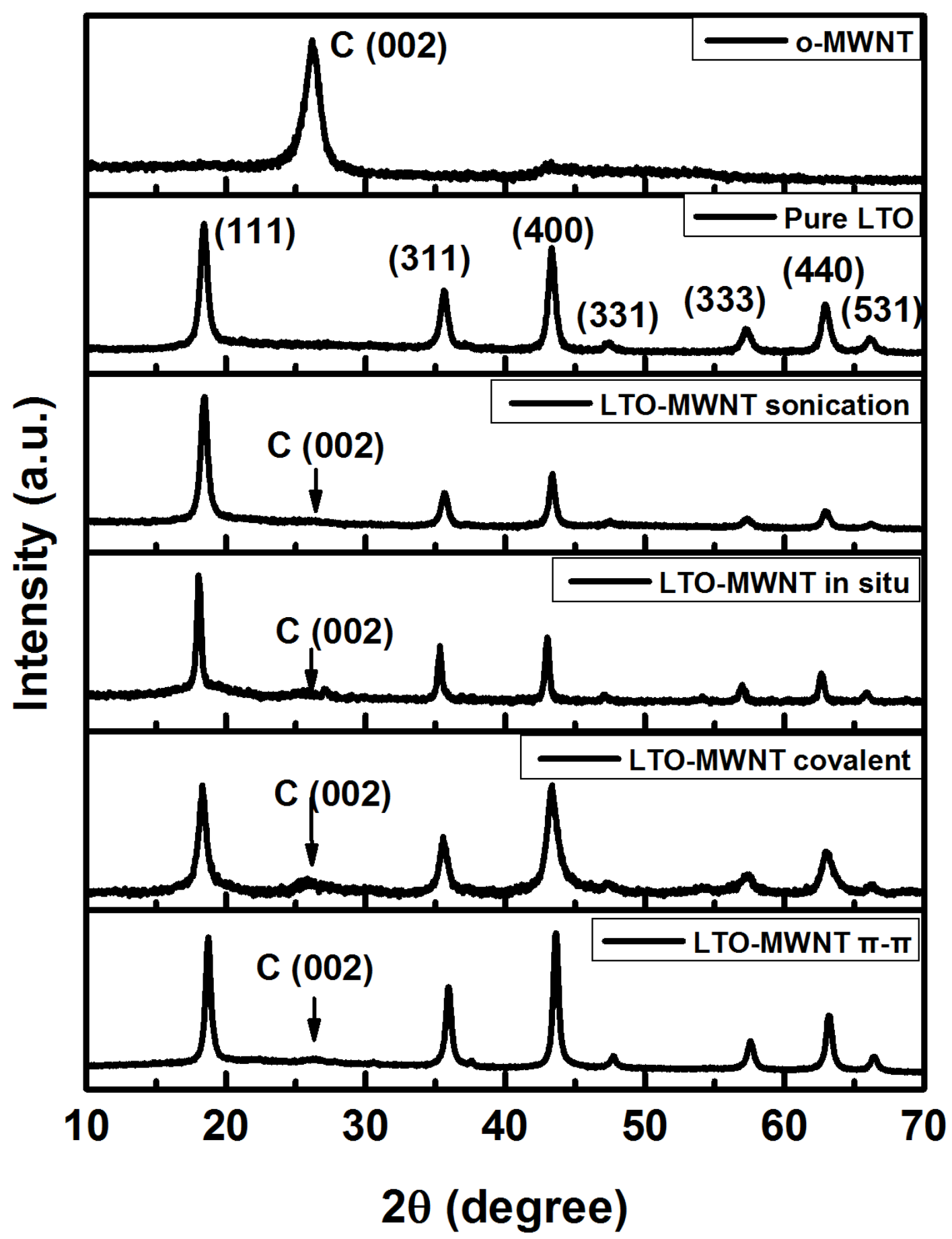

Figure 1. (Top to bottom) XRD patterns associated with (i) the oxidized MWNTs, (ii) pure flower-shape LTO motifs, and (iii) the resulting LTO-MWNT composites, generated by sonication, direct in situ, covalent attachment, and $\pi-\pi$ interaction strategies, respectively. 


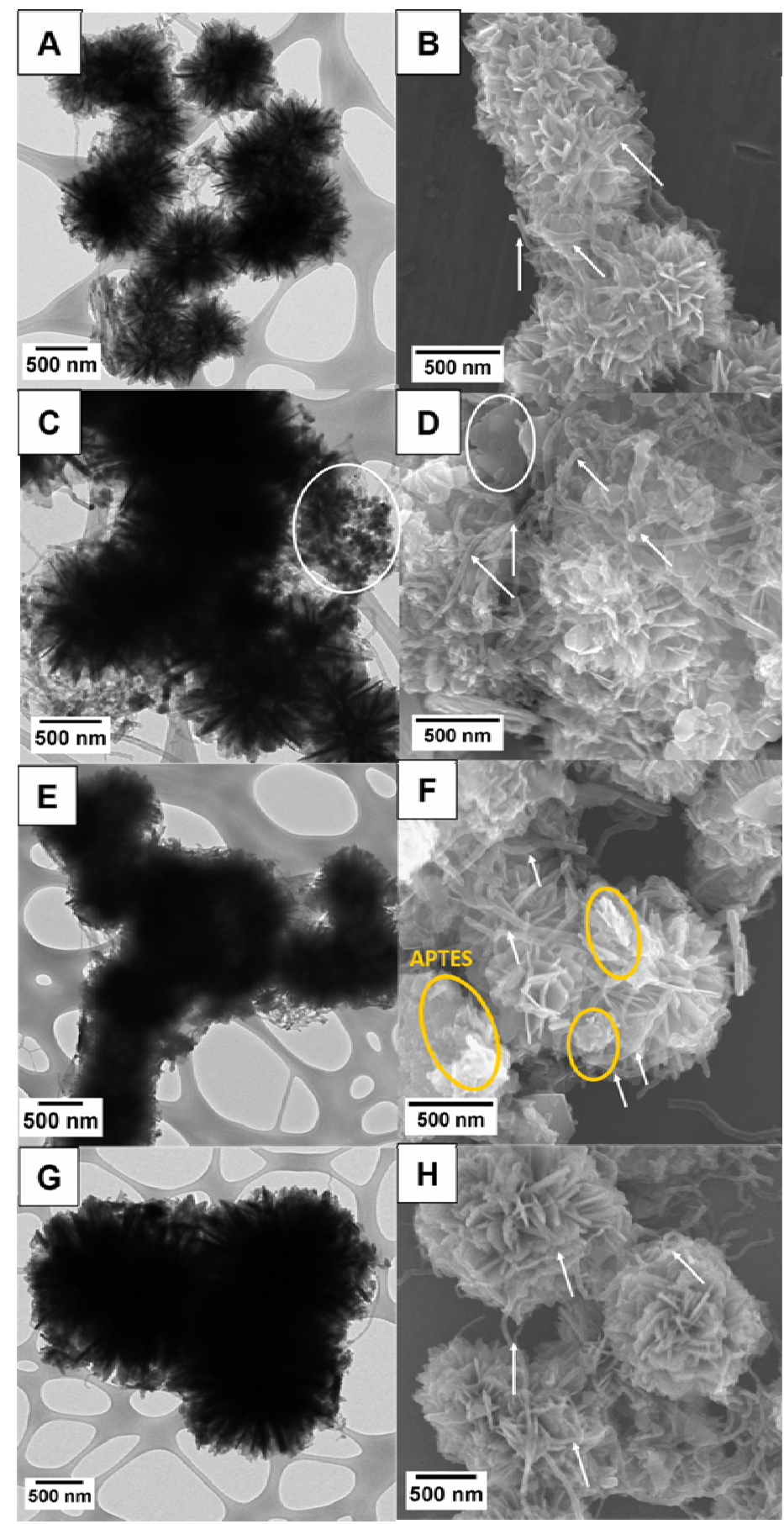

Figure 2. TEM and SEM images of LTO-MWNT composites generated by sonication (A and $\mathbf{B})$, direct in situ $(\mathbf{C}$ and $\mathbf{D})$, covalent attachment $(\mathbf{E}$ and $\mathbf{F})$, and $\pi-\pi$ interaction $(\mathbf{G}$ and $\mathbf{H}$ ) strategies, respectively. The MWNTs are indicated by the white arrows and the fragmented LTO structures are denoted by the white circles. The existence of APTES molecular linkers within the LTO-MWNT sample generated by covalent attachment strategy is indicated by the yellow circles, shown in Figure F. 


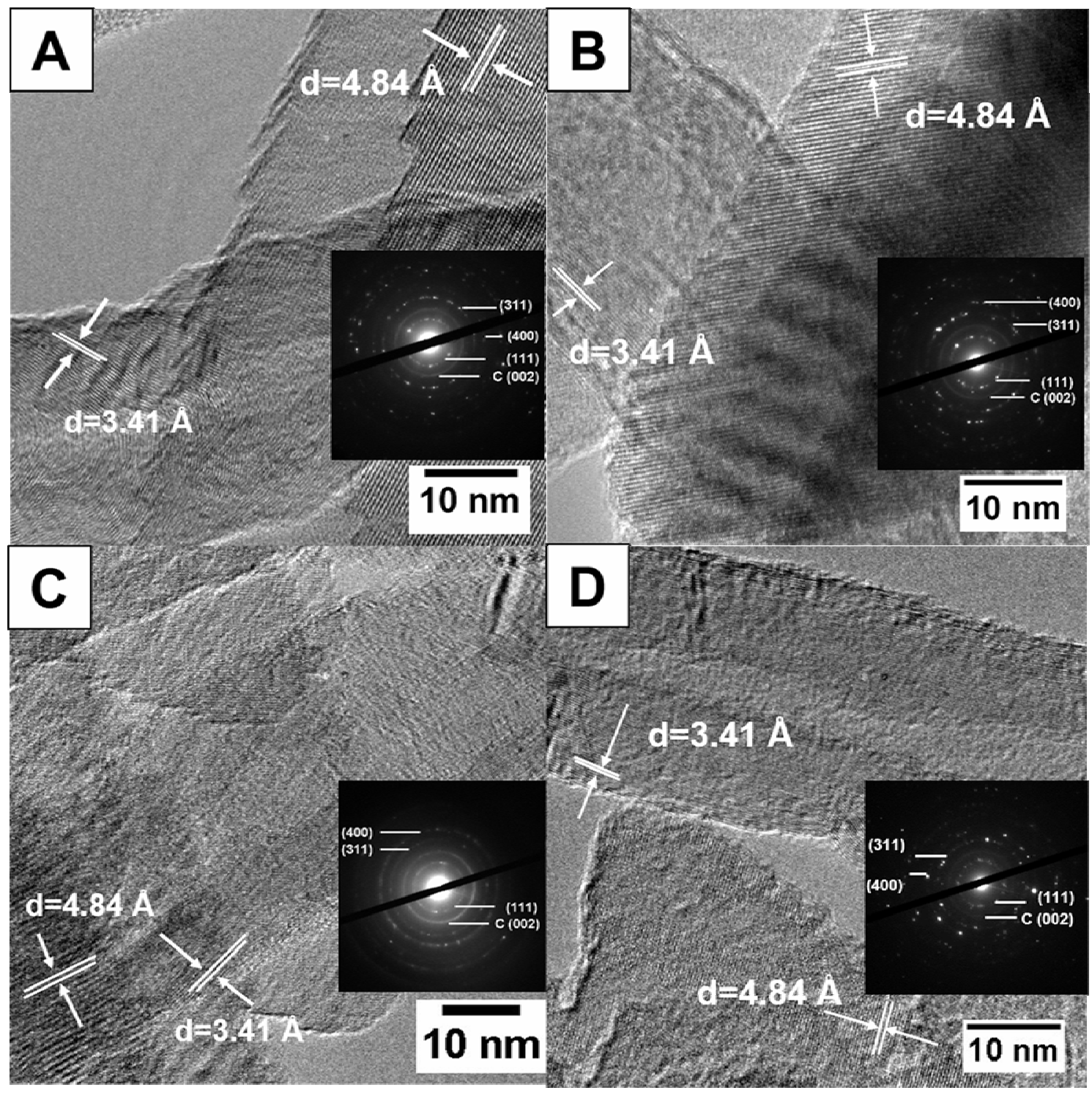

Figure 3. HRTEM images and the corresponding insets to the SAED patterns of LTOMWNT composites, generated by (A) physical sonication, (B) in situ growth, (C) covalent attachment, and (D) non-covalent $\pi-\pi$ interaction strategies, respectively. 


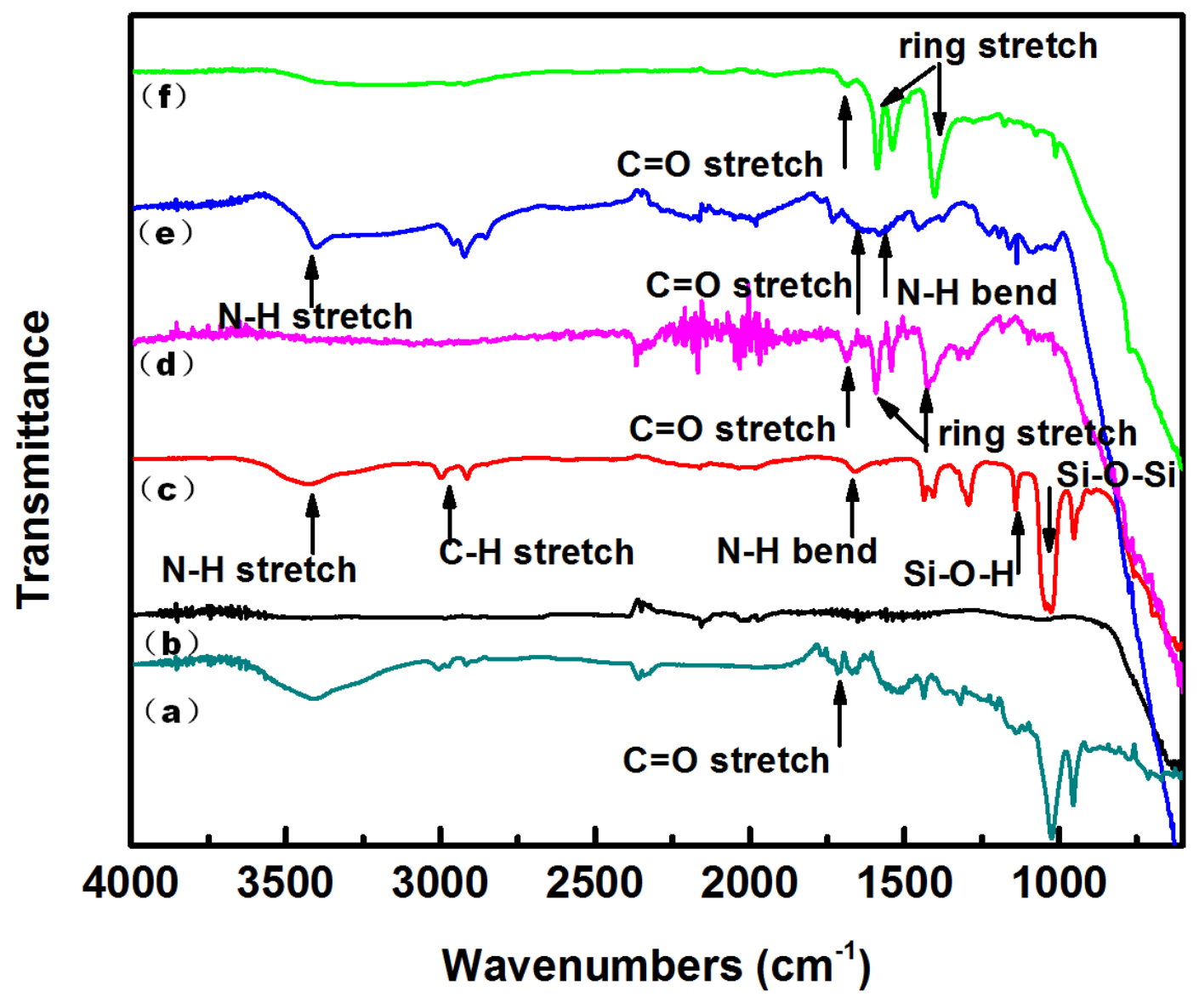

Figure 4. IR spectra of (a) oxidized MWNTs, (b) pure LTO, (c) APTES linkers, as well as (d) 4-MBA-functionalized LTO motifs, and associated LTO-MWNT heterostructures, generated not only by a (e) covalent attachment but also (f) $\pi-\pi$ interaction strategies. 

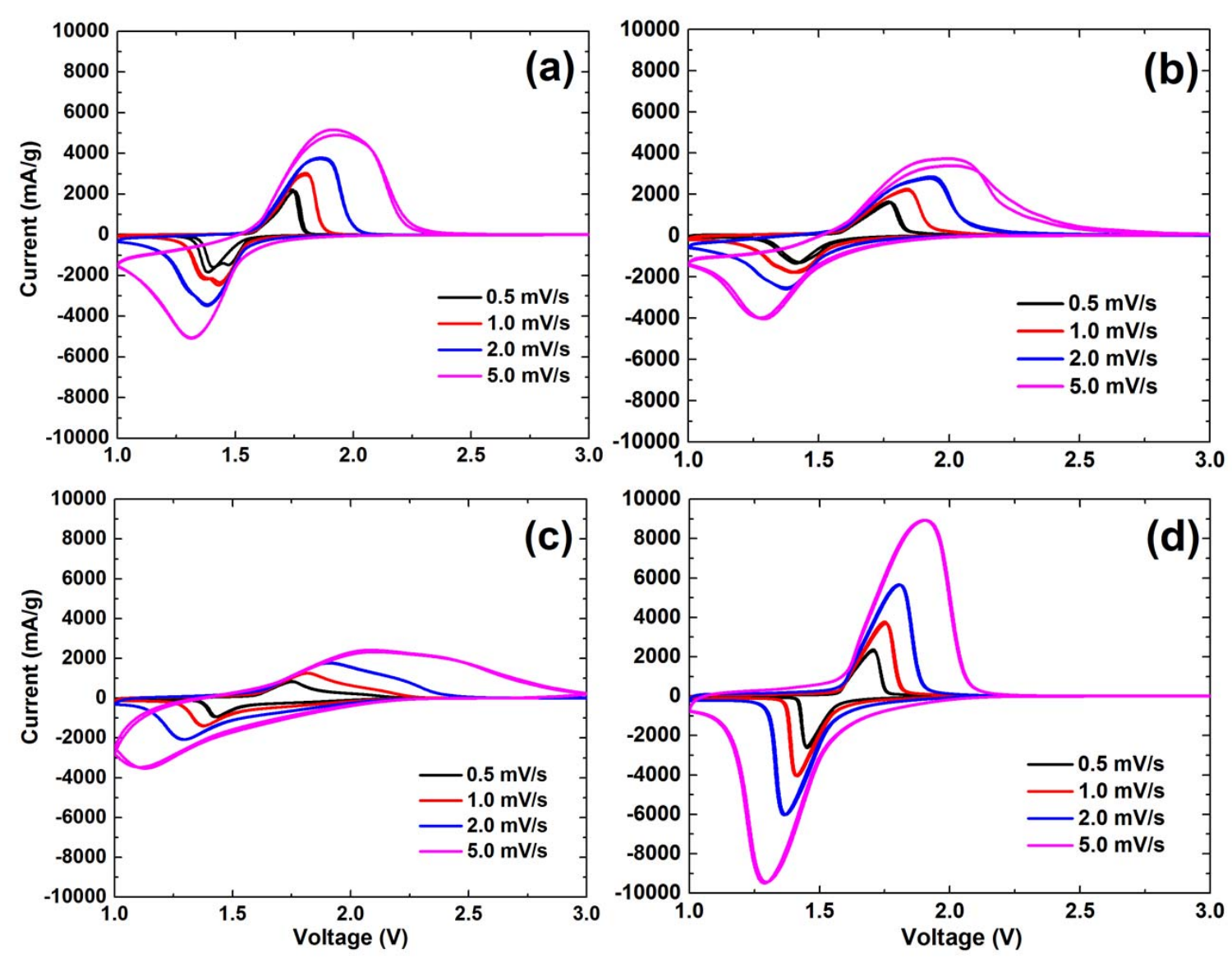

Figure 5. Cyclic voltammetry data obtained under scan rates of $0.5,1.0,2.0$, and 5.0 $\mathrm{mV} / \mathrm{s}$ of 2-electrode cells containing LTO-MWNT heterostructures generated by (a) in situ deposition, (b) sonication, (c) covalent attachment, and (d) non-covalent $\pi-\pi$ interaction strategies. 

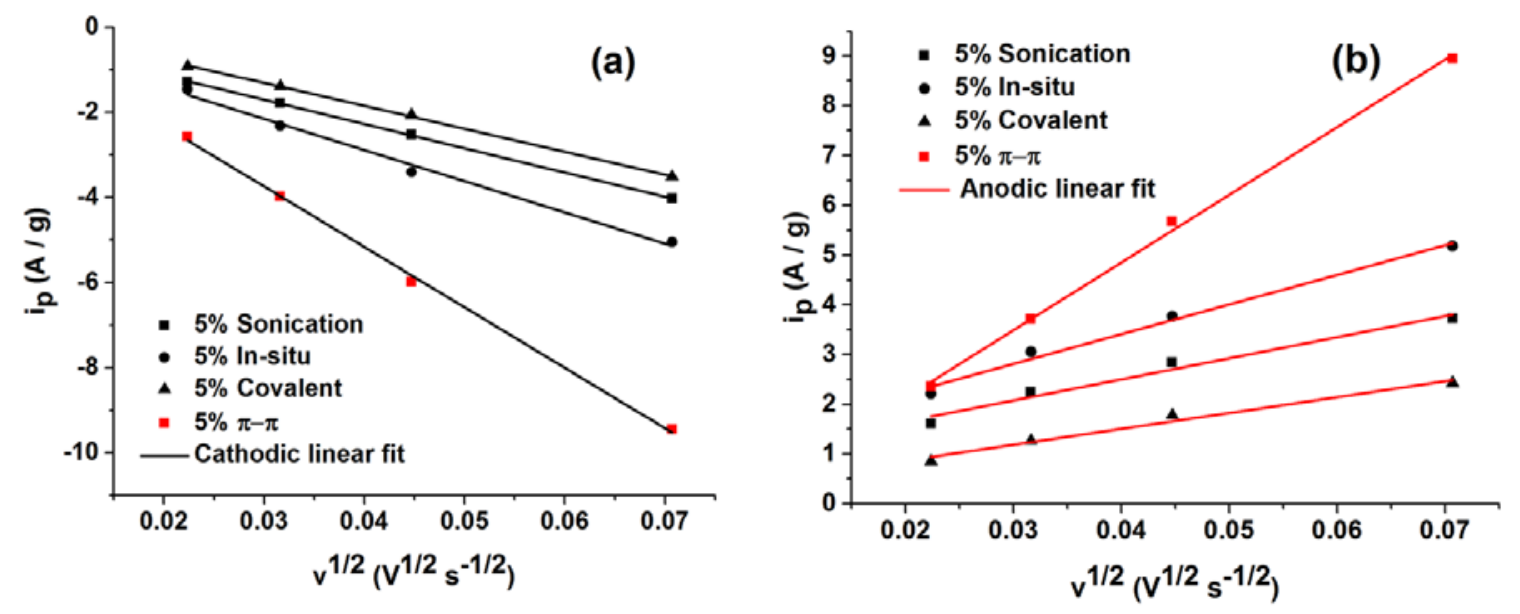

Figure 6. Fits of the CV data to the Randles-Sevcik equation, plots of $i_{p}$ as a function of square root of the scan rate for both (a) cathodic peaks and (b) anodic peaks. 

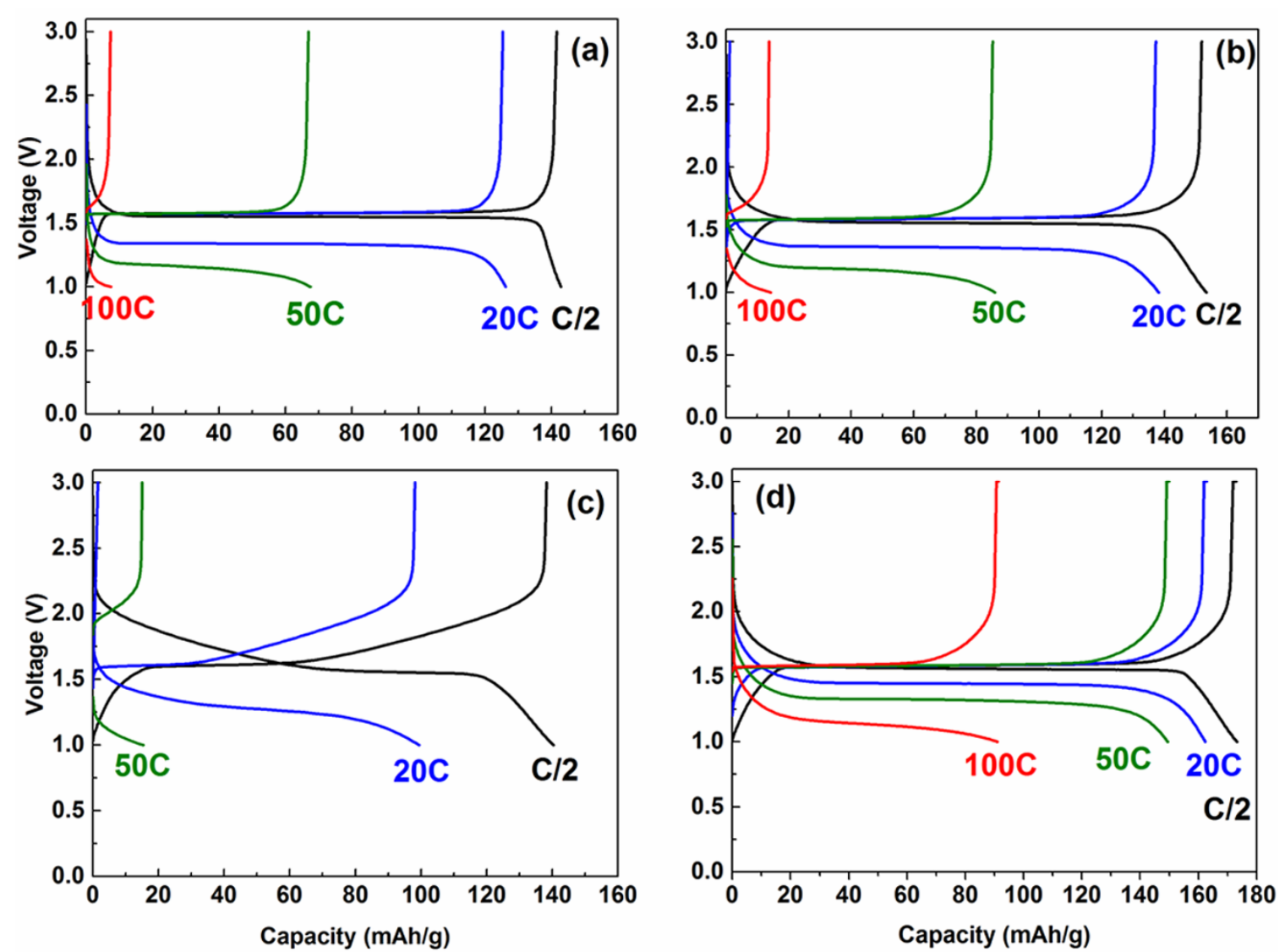

Figure 7. Discharge and charge voltage curves for lithium/LTO-MWNT electrochemical cells at cycle 20 ( $\mathrm{C} / 2$ rate), 25 (20 C rate), 30 (50 C rate), 35 (100 $\mathrm{C}$ rate). (a). Active material generated using a 5\% MWNT loading amount with the in situ method. (b). Active material fabricated using a 5\% MWNT loading amount with the physical sonication protocol. (c). Active material produced using a 5\% MWNT loading amount with covalent attachment. (d). Active material prepared using a 5\% MWNT loading amount synthesized through non-covalent $\pi$ - $\pi$ interactions. 


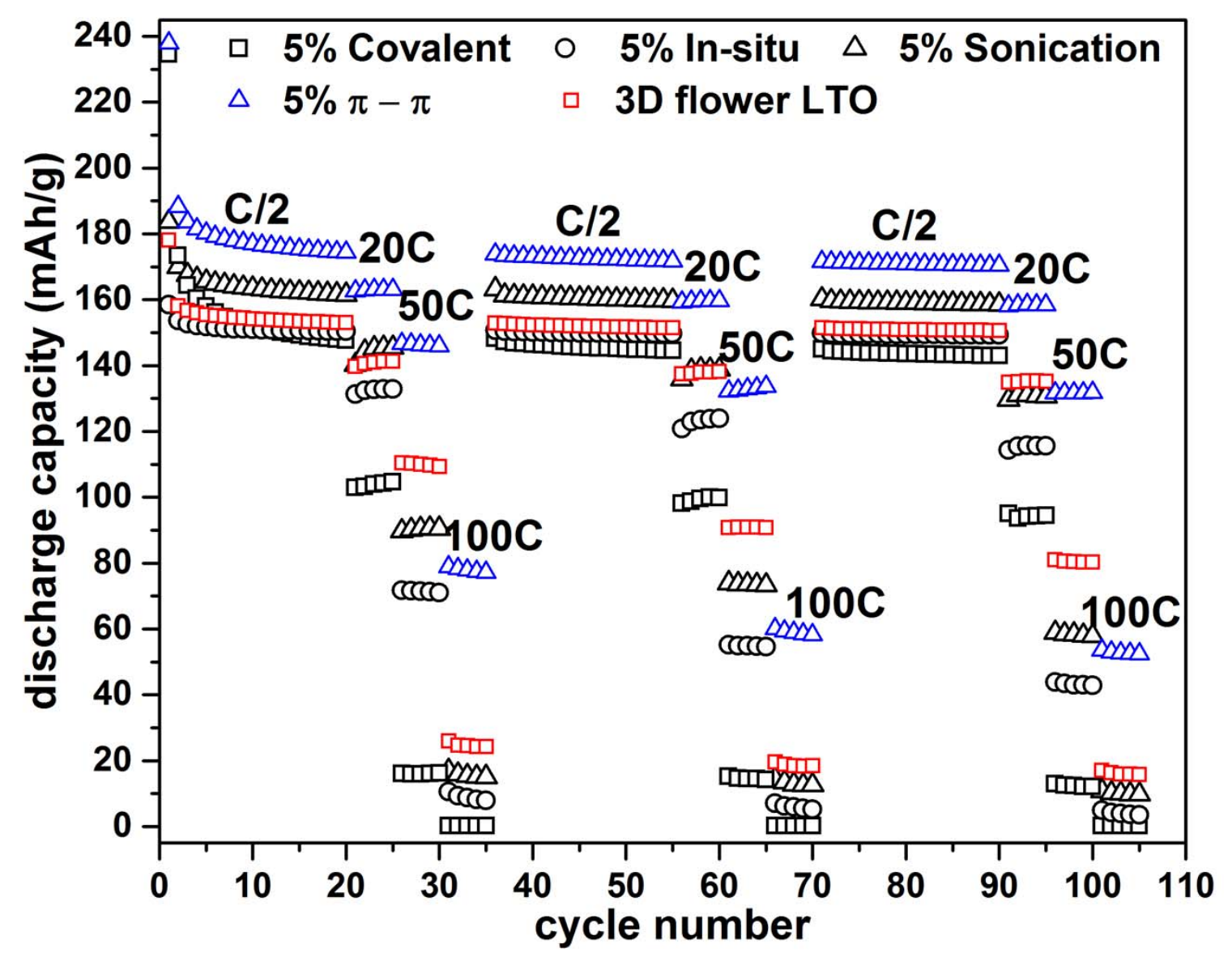

Figure 8. Discharge capacity versus cycle number for lithium/LTO-MWNT electrochemical cells created with active material composites prepared using sonication (black triangles), covalent attachment (black squares), in situ deposition (black circles), and $\pi-\pi$ interactions (blue triangles), respectively, at a 5\% MWNT loading level. The 3D flower LTO 'control' sample (red squares) tested in the same program is also shown here by means of comparison. 


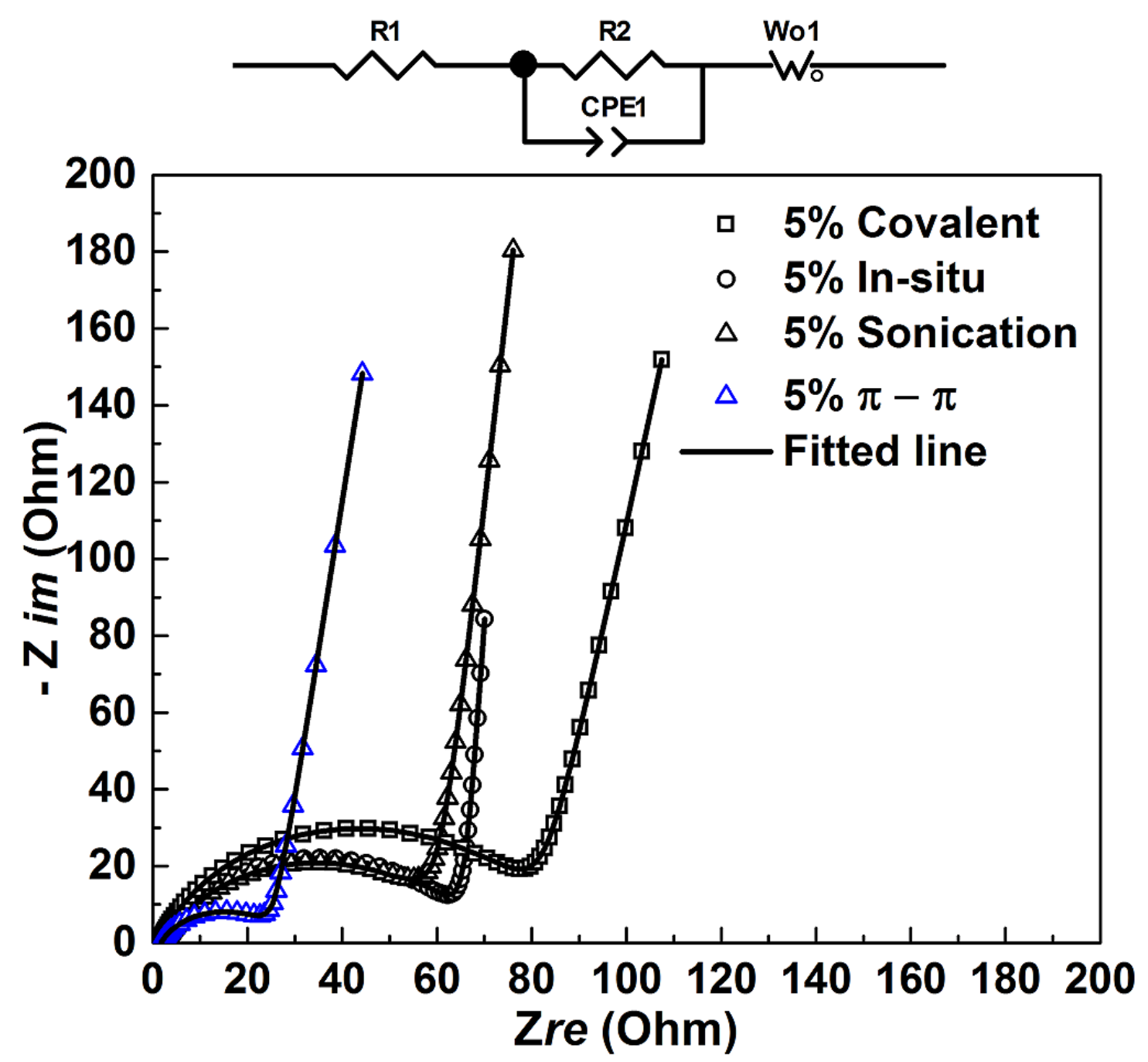

Figure 9. Equivalent circuit (top) and electrochemical impedance spectroscopy data (bottom) for lithium/LTO-MWNT electrochemical cells, incorporating active material composites fabricated using physical sonication (green triangles), covalent attachment (brown circles), in situ deposition (blue triangles), as well as $\pi$ - $\pi$ interaction (black squares) protocols, respectively, at a 5\% MWNT loading level. 


\section{Table of Contents Image}

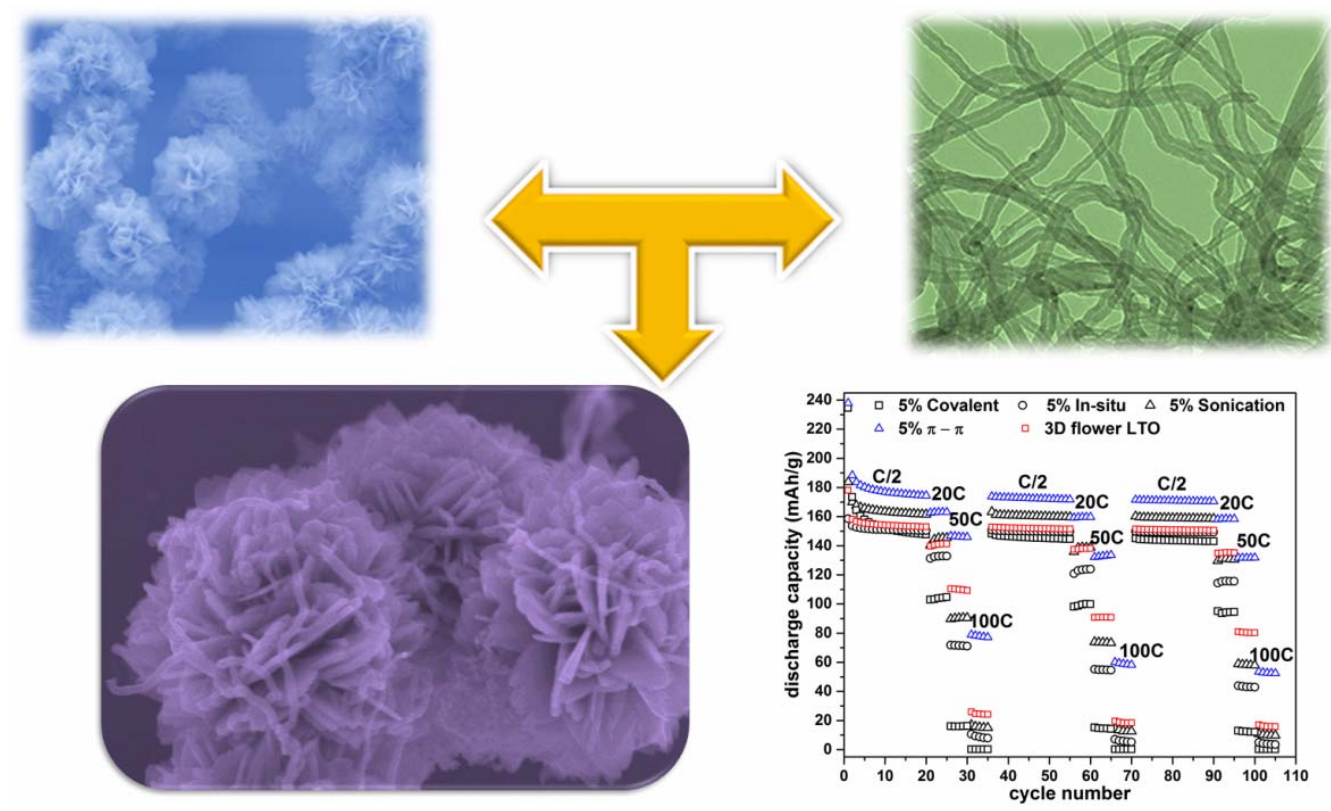

